\title{
Amplitude analysis of $D^{+} \rightarrow K_{S}^{0} \pi^{+} \pi^{+} \pi^{-}$
}

M. Ablikim, ${ }^{1}$ M. N. Achasov, ${ }^{10, d}$ S. Ahmed, ${ }^{15}$ M. Albrechtt ${ }^{4}$ M. Alekseev, ${ }^{55,55 c}$ A. Amoroso, ${ }^{55 a, 55 c}$ F. F. An, ${ }^{1}$ Q. An, ${ }^{42,52}$ Y. Bai ${ }^{41}$ O. Bakina, ${ }^{27}$ R. Baldini Ferroli, ${ }^{23 a}$ Y. Ban,${ }^{35}$ K. Begzsuren, ${ }^{25}$ J. V. Bennett, ${ }^{5}$ N. Berger, ${ }^{26}$ M. Bertani, ${ }^{23 a}$ D. Bettoni, ${ }^{24 a}$ F. Bianchi, ${ }^{55,55 \mathrm{c}}$ J. Bloms,${ }^{50}$ I. Boyko ${ }^{27}$ R. A. Briere,${ }^{5}$ H. Cai,${ }^{57}$ X. Cai,${ }^{1,42}$ A. Calcaterra, ${ }^{23 a}$ G. F. Cao, ${ }^{1,46}$ N. Cao, ${ }^{1,46}$ S. A. Cetin, ${ }^{45 b}$ J. Chai, ${ }^{55 c}$ J. F. Chang, ${ }^{1,42}$ W. L. Chang, ${ }^{1,46}$ G. Chelkov, ${ }^{27, b, c}$ Chen, ${ }^{6}$ G. Chen, ${ }^{1}$ H. S. Chen, ${ }^{1,46}$ J. C. Chen, ${ }^{1}$ M. L. Chen, ${ }^{1,42}$ S. J. Chen, ${ }^{33}$ Y. B. Chen, ${ }^{1,42}$ W. Cheng, ${ }^{55 c}$ G. Cibinetto, ${ }^{24 a}$ F. Cossio, ${ }^{55 c}$ X. F. Cui, ${ }^{34}$ H. L. Dai,${ }^{1,42}$ J. P. Dai, ${ }^{37, h}$ X. C. Dai,${ }^{1,46}$ A. Dbeyssi,${ }^{15}$ D. Dedovich, ${ }^{27}$ Z. Y. Deng, ${ }^{1}$ A. Denig,${ }^{26}$ I. Denysenko, ${ }^{27}$ M. Destefanis, ${ }^{55 a, 55 c}$ F. De Mori ${ }^{55 a, 55 c}$ Y. Ding, ${ }^{31}$ C. Dong, ${ }^{34}$ J. Dong, ${ }^{1,42}$ L. Y. Dong ${ }_{5,46}$ M. Y. Dong, ${ }^{1}$ Z. L. Dou, ${ }^{33}$ S. X. Du, ${ }^{60}$ J. Z. Fan, ${ }^{44}$ J. Fang, ${ }^{1,42}$ S. S. Fang, ${ }^{1,46}$ Y. Fang, ${ }^{1}$ R. Farinelli, ${ }^{24 a, 24 b}$ L. Fava, ${ }^{55 b, 55 c}$ F. Feldbauer, ${ }^{4}$ G. Felici, ${ }^{23 a}$ C. Q. Feng, ${ }^{42,52}$ M. Fritsch, ${ }^{4}$ C. D. Fu, ${ }^{1}$ Y. Fu, ${ }^{1}$ Q. Gao, ${ }^{1}$ X. L. Gao, ${ }^{42,52}$ Y. Gao, ${ }^{53}$ Y. Gao, ${ }^{44}$ Y. G. Gao, ${ }^{6}$ Z. Gao, ${ }^{42,52}$ B. Garillon, ${ }^{26}$ I. Garzia, ${ }^{24 a}$ A. Gilman, ${ }^{49}$ K. Goetzen, ${ }^{11}$ L. Gong,${ }^{34}$ W. X. Gong,,${ }^{1,42}$ W. Gradl, ${ }^{26}$ M. Greco, ${ }^{55 a}, 55 \mathrm{c}$ L. M. Gu, ${ }^{33}$ M. H. Gu, ${ }^{1,42}$ Y. T. Gu, ${ }^{13}$ A. Q. Guo, ${ }^{22}$ L. B. Guo, ${ }^{32}$ R. P. Guo, ${ }^{1,46}$ Y. P. Guo, ${ }^{26}$ A. Guskov, ${ }^{27}$ S. Han,${ }^{57}$ X. Q. Hao, ${ }^{16}$ F. A. Harris, ${ }^{47}$ K. L. He ${ }^{1,46}$ F. H. Heinsius, ${ }^{4}$ T. Held, ${ }^{4}$ Y. K. Heng, ${ }^{1}$ Y. R. Hou, ${ }^{46}$ Z. L. Hou, ${ }^{1}$ H. M. Hu, ${ }^{1,46}$ J. F. Hu, ${ }^{37, h}$ T. Hu, ${ }^{1}$ Y. Hu, ${ }^{1}$ G. S. Huang, ${ }^{42,52}$ J. S. Huang, ${ }^{16}$ X. T. Huang, ${ }^{36}$ X. Z. Huang, ${ }^{33}$ Z. L. Huang, ${ }^{31}$ T. Hussain,${ }^{54}$ N. Hsken, ${ }^{50}$ W. Ikegami Andersson, ${ }^{56}$ W. Imoehl, ${ }^{22}$ M. Irshad, ${ }^{42,52}$ Q. Ji, ${ }^{,}$Q. P. Ji, ${ }^{16}$ X. B. Ji, ${ }_{56}^{1,46}$ X. L. Ji, ${ }^{1,42}$ H. L. Jiang, ${ }^{36}$ X. S. Jiang, ${ }^{1}$ X. Y. Jiang, ${ }^{34}$ J. B. Jiao, ${ }^{36}$ Z. Jiao, ${ }^{18}$ D. P. Jin, ${ }^{1}$ S. Jin ${ }^{33}$ Y. Jin, ${ }^{48}$ T. Johansson,${ }^{56}$ N. Kalantar-Nayestanaki,${ }^{29}$ X. S. Kang, ${ }^{34}$ R. Kappert, ${ }^{29}$ M. Kavatsyuk, ${ }^{29}$ B. C. Ke ${ }^{1,61}$ I. K. Keshk, ${ }^{4}$ T. Khan, ${ }^{42,52}$ A. Khoukaz, ${ }^{50}$ P. Kiese, ${ }^{26}$ R. Kiuchi ${ }^{1}$ R. Kliemt, ${ }^{11}$ L. Koch, ${ }^{28}$ O. B. Kolcu, ${ }^{45 b, f}$ B. Kopf, ${ }^{4}$ M. Kuemmel, ${ }^{4}$ M. Kuessner, ${ }^{4}$ A. Kupsc, ${ }^{56}$ M. Kurth, ${ }^{1}$ M. G. Kurth, ${ }^{1,46}$ W. Kühn, ${ }^{28}$ J. S. Lange, ${ }^{28}$ P. Larin, ${ }^{15}$ L. Lavezzi, ${ }^{55 c, 1}$ H. Leithoff, ${ }^{26}$ T. Lenz, ${ }^{26}$ C. Li, ${ }^{56}$ Cheng Li, ${ }^{42,52}$ D. M. Li, ${ }^{60}$ F. Li ${ }^{1,42}$ F. Y. Li ${ }^{35}$ G. Li, ${ }^{1}$ H. B. Li ${ }^{1,46}$ H. J. Li,${ }^{9, j}$ J. C. Li, ${ }^{1}$ J. W. Li,${ }^{40}$ Ke Li ${ }^{1}$ L. K. Li,${ }^{1}{ }^{13}$ Lei Li,${ }^{3}$ P. L. Li ${ }^{42,52}$ P. R. Li, ${ }^{30}$ Q. Y. Li, ${ }^{36}$ W. D. Li ${ }^{1,46}$ W. G. Li, ${ }^{1}$ X. L. Li, ${ }^{36}$ X. N. Li,${ }^{1,42}$ X. Q. Li ${ }^{34}$ X. H. Li ${ }^{42,52}$ Z. B. Li, ${ }^{43}$ H. Liang, ${ }^{42,52}$ H. Liang, ${ }^{1,46}$ Y. F. Liang, ${ }^{39}$ Y. T. Liang, ${ }^{28}$ G. R. Liao, ${ }^{12}$ L. Z. Liao, ${ }^{1,46}$ J. Libby, ${ }^{21}$ C. X. Lin, ${ }^{43}$ D. X. Lin, ${ }^{15}$ Y. J. Lin ${ }^{13}{ }^{3}$ B. Liu, ${ }^{37, h}$ B. J. Liu,,${ }^{1}$ C. X. Liu ${ }^{1}$ D. Liu, ${ }^{4,52}$ D. Y. Liu ${ }^{37, h}$ F. H. Liu, ${ }^{38}$ Fang Liu, ${ }^{1}$ Feng Liu, ${ }^{6}$ H. B. Liu, ${ }^{13}$ H. M. Liu, ${ }^{1,46}$ Huanhuan Liu, ${ }^{1}$ Huihui Liu, ${ }^{17}$ J. B. Liu, ${ }^{42,52}$ J. Y. Liu, ${ }^{1,46}$ K. Y. Liu, ${ }^{31}$ Ke Liu, ${ }^{6}$ Q. Liu, ${ }^{46}$ S. B. Liu, ${ }^{42,52}$ T. Liu, ${ }^{1,46}$ X. Liu, ${ }^{30}$ X. Y. Liu, ${ }^{1,46}$ Y. B. Liu, ${ }^{34}$ Z. A. Liu, ${ }^{1}$ Zhiqing Liu, ${ }^{26}$ Y. F. Long, ${ }^{35}$ X. C. Lou, ${ }^{1}$ H. J. Lu, ${ }^{18}$ J. D. Lu, ${ }^{1,46}$ J. G. Lu, ${ }^{1,42}$ Y. Lu $\odot,{ }^{1}$ Y. P. Lu, ${ }^{1,42}$ C. L. Luo, ${ }^{32}$ M. X. Luo, ${ }^{59}$ P. W. Luo, ${ }^{43}$ T. Luo, ${ }^{9, j}$ X. L. Luo, ${ }^{1,42}$ S. Lusso, ${ }^{55 c}$ X. R. Lyu, ${ }^{46}$ F. C. Ma, ${ }^{31}$ H. L. Ma, ${ }^{1}$ L. L. Ma, ${ }^{36}$ M. M. Ma, ${ }^{1,46}$ Q. M. Ma ${ }^{1}$ X. N. Ma, ${ }^{34}$ X. X. Ma, ${ }^{1,46}$ X. Y. Ma, ${ }_{1}^{1,42}$ Y. M. Ma ${ }_{56}^{36}$ F. E. Maas, ${ }^{15}$ M. Maggiora, ${ }^{55 a, 55 c}$ S. Maldaner, ${ }^{26}$ Q. A. Malik, ${ }^{54}$

A. Mangoni, ${ }^{23 \mathrm{~b}}$ Y. J. Mao, ${ }^{35}$ Z. P. Mao, ${ }^{1}$ S. Marcello,${ }^{55 a, 55 \mathrm{c}}$ Z. X. Meng, ${ }^{48}$ J. G. Messchendorp, ${ }^{29}$ G. Mezzadri, ${ }^{24 \mathrm{a}}$ J. Min ${ }^{1,42}$

T. J. Min ${ }^{33}$ R. E. Mitchell ${ }^{22}$ X. H. Mo, ${ }^{1}$ Y. J. Mo, ${ }^{6}$ C. Morales Morales, ${ }^{15}$ N. Yu. Muchnoi, ${ }^{10, d}$ H. Muramatsu, ${ }^{49}$

A. Mustafa, ${ }^{4}$ S. Nakhoul, ${ }_{11, \mathrm{~g}}$ Y. Nefedov, ${ }^{27}$ F. Nerling, ${ }^{11, \mathrm{~g}}$ I. B. Nikolaev, ${ }^{10, \mathrm{~d}}$ Z. Ning ${ }^{1,42}$ S. Nisar, ${ }_{4}^{8, \mathrm{k}}$ S. L. Niu, ${ }^{1,42}$

S. L. Olsen, ${ }^{46}$ Q. Ouyang, ${ }^{1}$ S. Pacetti ${ }^{23 b}$ Y. Pan ${ }^{42,52}$ M. Papenbrock, ${ }^{56}$ P. Patteri, ${ }^{23 a}$ M. Pelizaeus, ${ }^{4}$ H. P. Peng, ${ }^{42,52}$

K. Peters ${ }^{11, g}$ J. Pettersson, ${ }^{56}$ J. L. Ping ${ }^{32}$ R. G. Ping, ${ }^{1,46}$ A. Pitka, ${ }^{4}$ R. Poling,${ }^{49}$ V. Prasad,${ }^{42,52}$ M. Qi,${ }^{33}$ T. Y. Qi,${ }^{2}$ S. Qian, ${ }^{1,42}$

C.F. Qiao, ${ }^{46}$ N. Qin ${ }^{57}$ X. P. Qin, ${ }^{13}$ X. S. Qin, ${ }_{5}{ }^{5}$ Z. H. Qin, ${ }^{1,42}$ J. F. Qiu, ${ }^{1}$ S. Q. Qu, ${ }^{34}$ K. H. Rashid,${ }^{54, i}$ C. F. Redmer, ${ }^{26}$ M. Richter, ${ }^{4}$ M. Ripka, ${ }^{26}$ A. Rivetti, ${ }^{55 c}$ M. Rolo,${ }^{55}$ G. Rong, ${ }^{1,46}$ Ch. Rosner, ${ }^{15}$ M. Rump, ${ }^{50}$ A. Sarantsev, ${ }^{27, \mathrm{e}}$ M. Savrié, ${ }^{24 \mathrm{~b}}$ K. Schoenning, ${ }^{56}$ W. Shan,${ }^{19}$ X. Y. Shan,${ }^{42,52}$ M. Shao, ${ }^{42,52}$ C. P. Shen, ${ }^{2}$ P. X. Shen, ${ }^{34}$ X. Y. Shen,,${ }^{1,46}$ H. Y. Sheng, ${ }^{1}$ X. Shi, ${ }^{1,42}$ X. D. Shi ${ }^{42,52}$ J. J. Song, ${ }^{36}$ Q. Q. Song, ${ }^{42,52}$ X. Y. Song, ${ }^{1}$ S. Sosio,${ }^{55,55 c}$ C. Sowa ${ }^{4}$ S. Spataro, ${ }^{55 a, 55 c}$ F. F. Sui ${ }^{36}$ G. X. Sun, ${ }^{1}$ J. F. Sun, ${ }^{16}$ L. Sun, ${ }^{37}$ S. S. Sun, ${ }^{1,46}$ X. H. Sun, ${ }^{1}$ Y. J. Sun, ${ }^{42,52}$ Y. K. Sun, ${ }^{42,52}$ Y. Z. Sun ${ }^{1}$ Z. J. Sun, ${ }^{1,42}$ Z. T. Sun, ${ }^{1}$ Y. T. Tan, ${ }^{42,52}$ C. J. Tang, ${ }^{39}$ G. Y. Tang, ${ }^{1}$ X. Tang,,${ }^{1}$ V. Thoren, ${ }^{56}$ B. Tsednee, ${ }^{25}$ I. Uman, ${ }^{45 d}$ B. Wang, ${ }^{1}$ B. L. Wang,${ }^{46}$ C. W. Wang ${ }_{1,46}^{33}$ D. Y. Wang, ${ }_{1}^{35}$ H. H. Wang ${ }_{58}^{36}$ K. Wang, ${ }^{1,42}$ L. L. Wang, ${ }^{1}$ L. S. Wang, ${ }^{1}$ M. Wang, ${ }^{36}$ M. Z. Wang, ${ }^{35}$ Meng Wang, ${ }^{1,46}$ P. L. Wang, ${ }^{1}$ R. M. Wang, ${ }^{58}$ W. P. Wang, ${ }^{42,52}$ X. Wang ${ }^{35}$ X. F. Wang, ${ }^{1}{ }^{15}$ Y. Wang, ${ }^{42,52}$ Y. F. Wang, ${ }^{1}$ Z. Wang, ${ }^{1,42}$ Z. G. Wang, ${ }^{1,42}$ Z. Y. Wang, ${ }^{1}$ Zongyuan Wang, ${ }^{1,46}$ T. Weber, ${ }^{4}$ D. H. Wei, ${ }^{12}$ P. Weidenkaff, ${ }^{26}{ }^{1}$ H. W. Wen, ${ }^{32}$ S. P. Wen, ${ }^{1}$ U. Wiedner, ${ }^{4}$ M. Wolke, ${ }^{56}$ L. H. Wu, ${ }^{1}$ L. J. Wu, ${ }^{1,46}$ Z. Wu, ${ }^{1,42}$ L. Xia, ${ }^{42,52}$ Y. Xia, ${ }^{20}$ S. Y. Xiao, ${ }^{1}$ Y. J. Xiao, ${ }_{1,46}^{1,46}$ Z. J. Xiao, ${ }^{32}$ Y. G. Xie, ${ }^{1,42}$ Y. H. Xie, ${ }^{6}$ T. Y. Xing, ${ }^{1,46}$ X. A. Xiong, ${ }^{1,46}$ Q. L. Xiu, ${ }^{1,42}$ G. F. Xu, ${ }^{1}{ }^{1}$ L. Xu, ${ }^{1}$ Q. J. Xu ${ }^{14}{ }^{14}$ W. Xu ${ }^{1,46}$ X. P. Xu, ${ }^{40}$ F. Yan, ${ }^{53}$ L. Yan, ${ }^{55,55 c}$ W. B. Yan, ${ }^{42,52}$ W. C. Yan, ${ }^{2}$ Y. H. Yan, ${ }^{20}$ H. J. Yang, ${ }^{37, h}$ H. X. Yang, ${ }^{1}$ L. Yang, ${ }^{57}$ R. X. Yang, ${ }^{42,52}$ S. L. Yang, ${ }^{1,46}$ Y. H. Yang, ${ }^{33}$ Y. X. Yang, ${ }_{12}^{12}$ Yifan Yang, ${ }^{1,46}$ Z. Q. Yang, ${ }^{20}$ M. Ye, ${ }^{1,42}$ M. H. Ye, ${ }^{7}$ J. H. Yin, ${ }^{1}$ Z. Y. You, ${ }^{43}$ B. X. Yu, ${ }^{1}$ C. X. Yu, ${ }^{34}$ J. S. Yu, ${ }^{20}$ C. Z. Yuan, ${ }^{1,46}$ X. Q. Yuan, ${ }^{35}$ Y. Yuan, ${ }^{1}$ A. Yuncu, ${ }^{45 b, a}$ A. A. Zafar, ${ }^{54}$ Y. Zeng, ${ }^{20}$ B. X. Zhang, ${ }^{1}$ B. Y. Zhang, ${ }^{1,42}$ C. C. Zhang, ${ }^{1}$ D. H. Zhang, ${ }_{1}^{1}$ H. H. Zhang, ${ }^{43}$ H. Y. Zhang, ${ }^{1,42}$ J. Zhang, ${ }^{1,46}$ J. L. Zhang, ${ }^{58}$ J. Q. Zhang, ${ }^{4}$ J. W. Zhang, ${ }^{1}$ J. Y. Zhang, ${ }_{1}$ J. Z. Zhang, ${ }^{1,46}$ K. Zhang, ${ }^{1,46}$ L. Zhang, ${ }^{44}$ S. F. Zhang, ${ }^{33}{ }^{4}$ T. J. Zhang, ${ }^{37, \mathrm{~h}}$ X. Y. Zhang, ${ }^{36}$ Y. Zhang, ${ }^{42,52}$ Y. H. Zhang, ${ }^{1,42}$ Y. T. Zhang, ${ }^{42,52}$ Yang Zhang, ${ }_{1,4 a o}$ Zhang, ${ }^{1}$ Yu Zhang, ${ }^{46}$ Z. H. Zhang, ${ }^{6}$ Z. P. Zhang, ${ }^{52}$ Z. Y. Zhang, ${ }^{57}$ G. Zhao, ${ }^{1}$ J. W. Zhao, ${ }^{1,42}$ J. Y. Zhao, ${ }^{1,46}$ J. Z. Zhao, ${ }^{1,42}$ Lei Zhao ${ }^{42,52}$ Ling Zhao, ${ }^{1}$ M. G. Zhao, ${ }^{34}$ Q. Zhao, ${ }^{1}$ S. J. Zhao, ${ }^{60}$ T. C. Zhao, ${ }^{1}$ Y. B. Zhao, ${ }^{1,42}$ Z. G. Zhao, ${ }^{42,52}$ A. Zhemchugov, ${ }^{27, b}$ B. Zheng, ${ }^{53}$ J. P. Zheng, ${ }^{1,42}$ Y. Zheng, ${ }^{35}$ Y. H. Zheng, ${ }^{46}$ B. Zhong, ${ }^{32}$ L. Zhou, ${ }^{1,42}$ L. P. Zhou, ${ }^{1,46}$ Q. Zhou, ${ }^{1,46}$ X. Zhou, ${ }^{57}$ X. K. Zhou, ${ }^{46}$ X. R. Zhou, ${ }^{42,52}$ 
Xiaoyu Zhou, ${ }^{20}$ Xu Zhou, ${ }^{20}$ A. N. Zhu, ${ }^{1,46}$ J. Zhu, ${ }^{34}$ J. Zhu, ${ }^{43}$ K. Zhu, ${ }^{1}$ K. J. Zhu, ${ }^{1}$ S. H. Zhu, ${ }^{51}$ W. J. Zhu, ${ }^{34}$ X. L. Zhu, ${ }^{44}$ Y. C. Zhu, ${ }^{42,52}$ Y. S. Zhu, ${ }^{1,46}$ Z. A. Zhu, ${ }^{1,46}$ J. Zhuang, ${ }^{1,42}$ B. S. Zou, ${ }^{1}$ and J. H. Zou ${ }^{1}$

(BESIII Collaboration)

${ }^{1}$ Institute of High Energy Physics, Beijing 100049, People's Republic of China

${ }^{2}$ Beihang University, Beijing 100191, People's Republic of China

${ }^{3}$ Beijing Institute of Petrochemical Technology, Beijing 102617, People's Republic of China

${ }^{4}$ Bochum Ruhr-University, D-44780 Bochum, Germany

${ }^{5}$ Carnegie Mellon University, Pittsburgh, Pennsylvania 15213, USA

${ }^{6}$ Central China Normal University, Wuhan 430079, People's Republic of China

${ }^{7}$ China Center of Advanced Science and Technology, Beijing 100190, People's Republic of China

${ }^{8}$ COMSATS University Islamabad, Lahore Campus, Defence Road, Off Raiwind Road, 54000 Lahore, Pakistan

${ }^{9}$ Fudan University, Shanghai 200443, People's Republic of China

${ }^{10}$ G.I. Budker Institute of Nuclear Physics SB RAS (BINP), Novosibirsk 630090, Russia

${ }^{11}$ GSI Helmholtzcentre for Heavy Ion Research GmbH, D-64291 Darmstadt, Germany

${ }^{12}$ Guangxi Normal University, Guilin 541004, People's Republic of China

${ }^{13}$ Guangxi University, Nanning 530004, People's Republic of China

${ }^{14}$ Hangzhou Normal University, Hangzhou 310036, People's Republic of China

${ }^{15}$ Helmholtz Institute Mainz, Johann-Joachim-Becher-Weg 45, D-55099 Mainz, Germany

${ }^{16}$ Henan Normal University, Xinxiang 453007, People's Republic of China

${ }^{17}$ Henan University of Science and Technology, Luoyang 471003, People's Republic of China

${ }^{18}$ Huangshan College, Huangshan 245000, People's Republic of China

${ }^{19}$ Hunan Normal University, Changsha 410081, People's Republic of China

${ }^{20}$ Hunan University, Changsha 410082, People's Republic of China

${ }^{21}$ Indian Institute of Technology Madras, Chennai 600036, India

${ }^{22}$ Indiana University, Bloomington, Indiana 47405, USA

${ }^{23 a}$ INFN Laboratori Nazionali di Frascati, I-00044, Frascati, Italy

${ }^{23 \mathrm{~b}}$ INFN and University of Perugia, I-06100, Perugia, Italy

${ }^{24 \mathrm{a}}$ INFN Sezione di Ferrara, I-44122, Ferrara, Italy

${ }^{24 \mathrm{~b}}$ University of Ferrara, I-44122, Ferrara, Italy

${ }^{25}$ Institute of Physics and Technology, Peace Ave. 54B, Ulaanbaatar 13330, Mongolia

${ }^{26}$ Johannes Gutenberg University of Mainz, Johann-Joachim-Becher-Weg 45, D-55099 Mainz, Germany

${ }^{27}$ Joint Institute for Nuclear Research, 141980 Dubna, Moscow region, Russia

${ }^{28}$ Justus-Liebig-Universitaet Giessen, II. Physikalisches Institut, Heinrich-Buff-Ring 16, D-35392 Giessen, Germany

${ }^{29}$ KVI-CART, University of Groningen, NL-9747 AA Groningen, The Netherlands

${ }^{30}$ Lanzhou University, Lanzhou 730000, People's Republic of China

${ }^{31}$ Liaoning University, Shenyang 110036, People's Republic of China

${ }^{32}$ Nanjing Normal University, Nanjing 210023, People's Republic of China

${ }^{33}$ Nanjing University, Nanjing 210093, People's Republic of China

${ }^{34}$ Nankai University, Tianjin 300071, People's Republic of China

${ }^{35}$ Peking University, Beijing 100871, People's Republic of China

${ }^{36}$ Shandong University, Jinan 250100, People's Republic of China

${ }^{37}$ Shanghai Jiao Tong University, Shanghai 200240, People's Republic of China

${ }^{38}$ Shanxi University, Taiyuan 030006, People's Republic of China

${ }^{39}$ Sichuan University, Chengdu 610064, People's Republic of China

${ }^{40}$ Soochow University, Suzhou 215006, People's Republic of China

${ }^{41}$ Southeast University, Nanjing 211100, People's Republic of China

${ }^{42}$ State Key Laboratory of Particle Detection and Electronics, Beijing 100049, Hefei 230026, People's Republic of China

${ }^{43}$ Sun Yat-Sen University, Guangzhou 510275, People's Republic of China

${ }^{44}$ Tsinghua University, Beijing 100084, People's Republic of China

${ }^{45 a}$ Ankara University, 06100 Tandogan, Ankara, Turkey

${ }^{45 \mathrm{~b}}$ Istanbul Bilgi University, 34060 Eyup, Istanbul, Turkey

${ }^{45 c}$ Uludag University, 16059 Bursa, Turkey

${ }^{45 \mathrm{~d}}$ Near East University, Nicosia, North Cyprus, Mersin 10, Turkey

${ }^{46}$ University of Chinese Academy of Sciences, Beijing 100049, People's Republic of China

${ }^{47}$ University of Hawaii, Honolulu, Hawaii 96822, USA 


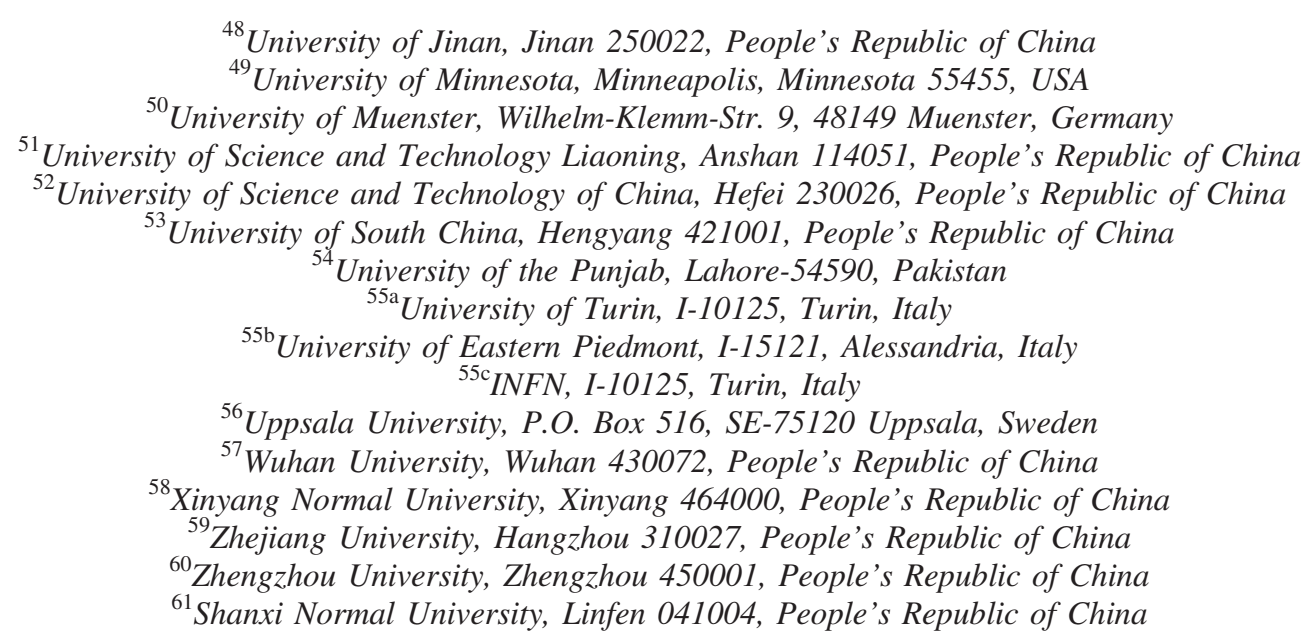

(Received 18 January 2019; published 18 October 2019; corrected 11 February 2021)

The decay $D^{+} \rightarrow K_{S}^{0} \pi^{+} \pi^{+} \pi^{-}$is studied with an amplitude analysis using a data set of $2.93 \mathrm{fb}^{-1}$ of $e^{+} e^{-}$ collisions at the $\psi(3770)$ peak accumulated by the BESIII detector. Intermediate states and nonresonant components, and their relative fractions and phases, have been determined. The significant amplitudes, which contribute to the model that best fits the data, are composed of five quasitwo-body decays $K_{S}^{0} a_{1}(1260)^{+}$, $\bar{K}_{1}(1270)^{0} \pi^{+} \bar{K}_{1}(1400)^{0} \pi^{+}, \bar{K}_{1}(1650)^{0} \pi^{+}$, and $\bar{K}(1460)^{0} \pi^{+}$, a three-body decay $K_{S}^{0} \pi^{+} \rho^{0}$, as well as a nonresonant component $K_{S}^{0} \pi^{+} \pi^{+} \pi^{-}$. The dominant amplitude is $K_{S}^{0} a_{1}(1260)^{+}$, with a fit fraction of $(40.3 \pm 2.1 \pm 2.9) \%$, where the first and second uncertainties are statistical and systematic, respectively.

DOI: $10.1103 /$ PhysRevD.100.072008

\section{INTRODUCTION}

${ }^{a}$ Also at Bogazici University, 34342 Istanbul, Turkey.

${ }^{\mathrm{b}}$ Also at the Moscow Institute of Physics and Technology, Moscow 141700, Russia.

${ }^{\mathrm{c}}$ Also at the Functional Electronics Laboratory, Tomsk State University, Tomsk, 634050, Russia.

${ }^{\mathrm{d}}$ Also at the Novosibirsk State University, Novosibirsk, 630090, Russia.

"Also at the NRC "Kurchatov Institute", PNPI, 188300, Gatchina, Russia.

${ }^{\mathrm{f}}$ Also at Istanbul Arel University, 34295 Istanbul, Turkey.

${ }^{\mathrm{g}}$ Also at Goethe University Frankfurt, 60323 Frankfurt am Main, Germany.

${ }^{\mathrm{h}}$ Also at Key Laboratory for Particle Physics, Astrophysics and Cosmology, Ministry of Education; Shanghai Key Laboratory for Particle Physics and Cosmology; Institute of Nuclear and Particle Physics, Shanghai 200240, People's Republic of China.

${ }^{1}$ Also at Government College Women University, Sialkot51310 Punjab, Pakistan.

${ }^{\mathrm{j}}$ Also at Key Laboratory of Nuclear Physics and Ion-beam Application (MOE) and Institute of Modern Physics, Fudan University, Shanghai 200443, People's Republic of China.

${ }^{\mathrm{k}}$ Also at Harvard University, Department of Physics, Cambridge, Massachusetts 02138, USA.

Published by the American Physical Society under the terms of the Creative Commons Attribution 4.0 International license. Further distribution of this work must maintain attribution to the author(s) and the published article's title, journal citation, and DOI. Funded by SCOAP ${ }^{3}$.
Hadronic decays of mesons with charm are an important tool for understanding the dynamics of the strong interaction in the low energy regime. The amplitudes describing $D$ meson weak decays into four-body final states are dominated by (quasi-) two-body processes, such as $D \rightarrow V P, D \rightarrow S P, D \rightarrow V V$, and $D \rightarrow A P$, where $P, V, S$, and $A$ denote pseudoscalar, vector, scalar, and axial-vector mesons, respectively. Final-state interactions can cause significant changes in decay rates and shifts in the phases of decay amplitudes. Experimental measurements can help to refine theoretical models of these phenomena [1-3]. Many measurements on $D \rightarrow P P$ and $D \rightarrow V P$ decays have been performed [4]. However, there are only a few studies focusing on $D \rightarrow A P$ decays [4]. We have therefore measured $D \rightarrow A P$ decays via an amplitude analysis of the decay $D^{+} \rightarrow K_{S}^{0} \pi^{+} \pi^{+} \pi^{-}$(the inclusion of charge conjugate reaction is implied throughout the paper), which is expected to be dominated by $D^{+} \rightarrow K_{S}^{0} a_{1}(1260)^{+}$. In addition, the measurements of the intermediate processes containing $K_{1}(1270)$ and $K_{1}(1400)$ are helpful for understanding the mixture between these two axial-vector kaons [3].

In this paper, we present an amplitude analysis of the decay $D^{+} \rightarrow K_{S}^{0} \pi^{+} \pi^{+} \pi^{-}$to study the resonant substructures and nonresonant components, where the 
amplitude model is constructed using the covariant tensor formalism [5].

\section{DETECTOR AND DATA SETS}

The data used in this analysis were accumulated with the BESIII detector [6]. The event sample is based on $2.93 \mathrm{fb}^{-1}$ of $e^{+} e^{-}$collisions at the $\psi(3770)$ mass [7,8]. At this energy, $D$ meson pairs are produced without any additional hadrons. To suppress backgrounds from other charmed meson decays and continuum (QED process and light quark productions), only the decay mode $D^{-} \rightarrow K^{+} \pi^{-} \pi^{-}$is used to tag the $D^{+} D^{-}$pairs. This provides a clean environment for selecting the decay $D^{+} \rightarrow K_{S}^{0} \pi^{+} \pi^{+} \pi^{-}$ (the signal side) by requiring the $D^{-} \rightarrow K^{+} \pi^{-} \pi^{-}$decay to be observed (the tag side).

The BESIII detector located at Beijing Electron Positron Collider [9] is described in Ref. [6]. The geometrical acceptance of the BESIII detector is $93 \%$ of the full solid angle. Starting from the interaction point (IP), it consists of a main drift chamber (MDC), a time-of-flight (TOF) system, and a $\mathrm{CsI}(\mathrm{Tl})$ electromagnetic calorimeter, which are all enclosed in a superconducting solenoidal magnet providing a $1.0 \mathrm{~T}$ magnetic field. The solenoid is supported by an octagonal flux-return yoke with resistive plate counter muon identifier modules interleaved with steel. The momentum resolution for charged tracks in the MDC is $0.5 \%$ at a transverse momentum of $1 \mathrm{GeV} / c$. The energy resolution for the photon in electromagnetic calorimeter measurement is $2.5 \%(5 \%)$ in the barrel (end caps) region at $1 \mathrm{GeV}$. The time resolution of the TOF barrel part is $68 \mathrm{ps}$, while that of the end cap part is 110 ps.

Monte Carlo (MC) simulations of the BESIII detector are based on GEANT4 [10]. The production of $\psi(3770)$ is simulated with the KKMC [11] package, taking into account the beam energy spread and the initial-state radiation (ISR). The Pнотоs [12] package is used to simulate the final-state radiation of charged particles. The EVTGEN [13] package is used to simulate the known decay modes with branching fractions (BFs) taken from the Particle Data Group (PDG) [4], and the remaining unknown decays are generated with the LUNDCHARM model [14]. The MC sample referred to as "generic MC," including the processes of $\psi(3770)$ decays to $D \bar{D}$, non- $D \bar{D}$, ISR production of low mass charmonium states and continuum processes, is used to study the background contribution. The effective luminosities of the generic MC samples correspond to at least five times the data sample luminosity. Two kinds of MC samples with the decay chain $\psi(3770) \rightarrow D^{+} D^{-}$with $D^{+} \rightarrow K_{S}^{0} \pi^{+} \pi^{+} \pi^{-}$ and $D^{-} \rightarrow K^{+} \pi^{-} \pi^{-}$using different decay models are generated for the amplitude analysis. One sample, "PHSP MC," is generated with a uniform distribution in phase space for the $D^{+} \rightarrow K_{S}^{0} \pi^{+} \pi^{+} \pi^{-}$decay, which is used to calculate the $\mathrm{MC}$ integrations. The other sample, "signal $\mathrm{MC}$," is generated according to the results obtained in this analysis for the $D^{+} \rightarrow K_{S}^{0} \pi^{+} \pi^{+} \pi^{-}$decay. It is used to validate the fit performance, calculate the goodness of fit and estimate the detector efficiency.

\section{EVENT SELECTION}

Good charged tracks other than $K_{S}^{0}$ daughters are required to have a point of closest approach to the IP within $10 \mathrm{~cm}$ along the beam axis and within $1 \mathrm{~cm}$ in the plane perpendicular to the beam. The polar angle $\theta$ between the track and the $e^{+}$beam direction is required to satisfy $|\cos \theta|<0.93$. Separation of charged kaons from charged pions is implemented by combining the energy loss $(d E / d x)$ in the MDC and the time-of-fight information from the TOF. We calculate the probabilities $P(K)$ and $P(\pi)$ with the hypothesis of $K$ or $\pi$, and require that $K$ candidates have $P(K)>P(\pi)$, while $\pi$ candidates have $P(\pi)>P(K)$. Tracks without particle identification (PID) information are rejected. Furthermore, a vertex fit with the hypothesis that all tracks originate from the IP is performed, and the $\chi^{2}$ of the fit is required to be less than 100 .

The $K_{S}^{0}$ candidates are reconstructed from a pair of oppositely charged tracks which satisfy $|\cos \theta|<0.93$ and whose distances to the IP along the beam direction are within $20 \mathrm{~cm}$. The two charged tracks are assumed to be a $\pi^{+} \pi^{-}$pair without PID. In order to improve the signal-tobackground ratio, the decay vertex of the $\pi^{+} \pi^{-}$pair is required to be more than two standard deviations away from the IP [15], and their invariant mass is required to be in the region $[467.6,527.6] \mathrm{MeV} / c^{2}$.

The $D^{+} D^{-}$pair with $D^{+} \rightarrow K_{S}^{0} \pi^{+} \pi^{+} \pi^{-}$and $D^{-} \rightarrow$ $K^{+} \pi^{-} \pi^{-}$is reconstructed with the requirement that they do not have any tracks in common. If there are multiple $D^{+} D^{-}$candidates reconstructed in an event, the one with the average invariant mass closest to the nominal $D^{ \pm}$mass [4] is selected. To characterize the $D$ candidates, two variables, $M_{\mathrm{BC}}$ and $\Delta E$, defined as

$$
M_{\mathrm{BC}}=\sqrt{E_{\mathrm{beam}}^{2}-\vec{p}_{D}^{2}}
$$

and

$$
\Delta E=E_{D}-E_{\text {beam }},
$$

are calculated, where $\left(E_{D}, \vec{p}_{D}\right)$ is the reconstructed fourmomentum of $D$ candidate, and $E_{\text {beam }}$ is the calibrated beam energy. The signal events form a peak around 0 in the $\Delta E$ distribution and around the charged $D$ mass in the $M_{\mathrm{BC}}$ distribution. Events are required to satisfy $-0.027<$ $\Delta E\left(D_{\text {tag }}\right)<0.025 \mathrm{GeV},-0.033<\Delta E\left(D_{\text {signal }}\right)<0.030 \mathrm{GeV}$, and $1.8628<M_{\mathrm{BC}}<1.8788 \mathrm{GeV} / c^{2}$ for both tag and signal $D$ candidates. Figures $1(\mathrm{a})$ and $1(\mathrm{~b})$ show the two-dimensional (2D) distributions of tag side versus signal side for $\Delta E$ and $M_{\mathrm{BC}}$ of the accepted candidates, respectively. 

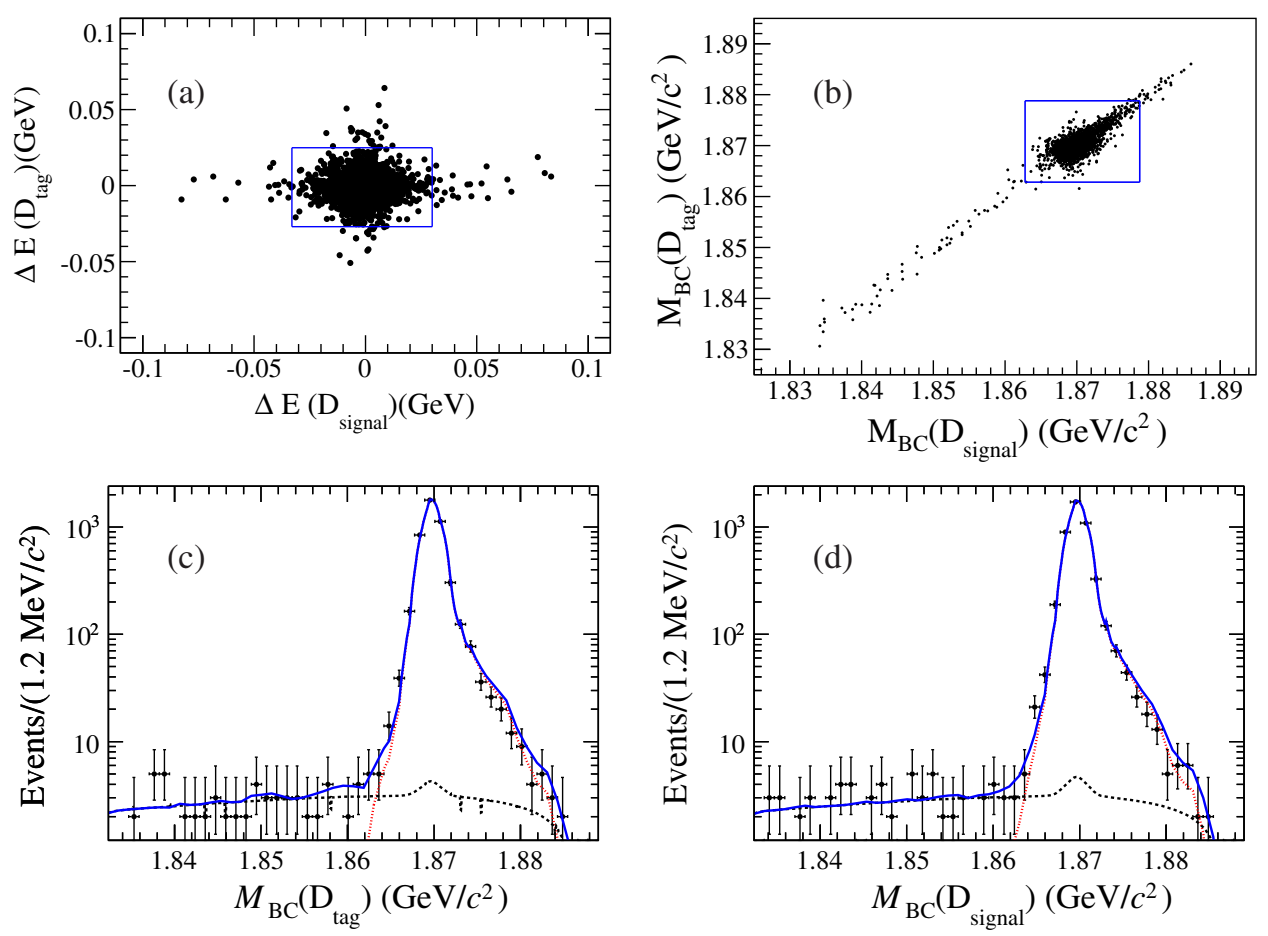

FIG. 1. Two-dimensional (a) $\Delta E$ (b) $M_{\mathrm{BC}}$ data distributions, and [(c) and (d)] fit $M_{\mathrm{BC}}$ projections. In (a) and (b), the rectangles shows the signal regions. In (c) and (d), data are compared with the projection (solid curve) of the 2D fit, with the signal and the background marked as the dotted and dashed curves, respectively. The small bump under the signal (tag) peak comes from the events with signal (tag) candidates properly reconstructed but tag (signal) candidates improperly reconstructed.

In order to suppress the peaking background of $D^{+} \rightarrow$ $K_{S}^{0} K_{S}^{0} \pi^{+}$with an additional $K_{S}^{0} \rightarrow \pi^{+} \pi^{-}$, which has the same final state as our signal decay, we perform a decay vertex constrained fit on any remaining $\pi^{+} \pi^{-}$pair with invariant mass within $\pm 30 \mathrm{MeV} / c^{2}$ of the mass of the $K_{S}^{0}$. The events are removed if the obtained decay length is greater than twice its uncertainty. After applying all selection criteria, the expected yield from the background $D^{+} \rightarrow K_{S}^{0} K_{S}^{0} \pi^{+}$is estimated to be $72.9 \pm 8.5$ by using the generic MC sample. In the amplitude analysis, it is subtracted by giving negative weights to the background events, as discussed in Sec. IVA. Self cross-feed events with misreconstructed signal decays are estimated from signal MC samples to be $\sim 0.1 \%$. This effect is considered as a systematic uncertainty.

To estimate the contribution from the nonpeaking background, a 2D unbinned maximum likelihood fit is performed to the $M_{\mathrm{BC}}\left(D_{\mathrm{tag}}\right)$ versus $M_{\mathrm{BC}}\left(D_{\text {signal }}\right)$ distribution in Fig. 1(c). The signal shape is modeled with the shape extracted from MC-simulated events. The function used to describe the diagonal background band is the product of an ARGUS function [16] in the $M_{\mathrm{BC}}\left(D_{\text {tag }}\right)+M_{\mathrm{BC}}\left(D_{\text {signal }}\right)$ plane and a Gaussian in the $M_{\mathrm{BC}}\left(D_{\text {tag }}\right)-M_{\mathrm{BC}}\left(D_{\text {signal }}\right)$ plane. The background with only the tag candidate (signal candidate) properly reconstructed peaks at the charged $D$ mass and spreads out on the other axis, which is parametrized as the product of a MC-simulated shape in
$M_{\mathrm{BC}}\left(D_{\text {tag }}\right)\left[M_{\mathrm{BC}}\left(D_{\text {signal }}\right)\right]$ and an ARGUS function on the other axis. The number of background events within the signal region extracted from the fit is $37.5 \pm 7.5$. The projection on $M_{\mathrm{BC}}\left(D_{\text {signal }}\right)$ from the $2 \mathrm{D}$ fit is shown in Fig. 1(d). The small background bump under the signal is from the events with the $D_{\text {signal }}$ properly reconstructed but the $D_{\text {tag }}$ improperly reconstructed. In the amplitude analysis, the general background is ignored and its effect is considered as a systematic uncertainty.

To improve the momentum resolution and ensure that all events fall within the phase space boundary, the selected candidate events are further subjected to a six-constraint (6C) kinematic fit. It constrains the total four-momentum of all final state particles to the initial four-momentum of the $e^{+} e^{-}$system, the invariant mass of signal side $D^{+} \rightarrow$ $K_{S}^{0} \pi^{+} \pi^{+} \pi^{-}$constrains to the $D^{+}$nominal mass, and the $K_{S}^{0}$ invariant mass constrains to the $K_{S}^{0}$ nominal mass. We discard events with a $\chi^{2}$ of $6 \mathrm{C}$ kinematic fit larger than 100 . After applying all selection criteria, 4559 candidate events are obtained with a purity of $97.5 \%$.

\section{AMPLITUDE ANALYSIS}

The goal of this analysis is to determine the intermediate components in the four-body $D^{+} \rightarrow K_{S}^{0} \pi^{+} \pi^{+} \pi^{-}$decay. The decay modes that may contribute to the $D^{+} \rightarrow K_{S}^{0} \pi^{+} \pi^{+} \pi^{-}$ decay are listed in Table I. The letters $S, D$ in square 
TABLE I. Spin factors $S(p)$ for different decay modes.

\begin{tabular}{lc}
\hline \hline Decay mode & $S(p)$ \\
\hline$D \rightarrow \mathrm{AP}_{1}, \mathrm{~A}[S] \rightarrow \mathrm{VP}_{2}, \mathrm{~V} \rightarrow \mathrm{P}_{3} \mathrm{P}_{4}$ & $\tilde{T}_{1}^{\mu}(D) P_{\mu \nu}^{(1)}(\mathrm{A}) \tilde{t}^{(1) \nu}(\mathrm{V})$ \\
$D \rightarrow \mathrm{AP}_{1}, \mathrm{~A}[D] \rightarrow \mathrm{VP}_{2}, \mathrm{~V} \rightarrow \mathrm{P}_{3} \mathrm{P}_{4}$ & $\tilde{T}^{(1) \mu}(D) \tilde{t}_{\mu \nu}^{(2)}(\mathrm{A}) \tilde{t}^{(1) \nu}(\mathrm{V})$ \\
$D \rightarrow \mathrm{AP}_{1}, \mathrm{~A} \rightarrow \mathrm{SP}_{2}, \mathrm{~S} \rightarrow \mathrm{P}_{3} \mathrm{P}_{4}$ & $\tilde{T}^{(1) \mu}(D) \tilde{t}_{\mu}^{(1)}(\mathrm{A})$ \\
$D \rightarrow \mathrm{V}_{1} \mathrm{P}_{1}, \mathrm{~V}_{1} \rightarrow \mathrm{V}_{2} \mathrm{P}_{2}, \mathrm{~V}_{2} \rightarrow \mathrm{P}_{3} \mathrm{P}_{4}$ & $\epsilon_{\mu \nu \lambda \sigma} p_{\mathrm{V}_{1}}^{\mu} q_{\mathrm{V}_{1}}^{\nu} p_{\mathrm{P}_{1}}^{\lambda} q_{\mathrm{V}_{2}}^{\sigma}$ \\
$D \rightarrow \mathrm{PP}_{1}, \mathrm{P} \rightarrow \mathrm{VP}_{2}, \mathrm{~V} \rightarrow \mathrm{P}_{3} \mathrm{P}_{4}$ & $p^{\mu}\left(\mathrm{P}_{2}\right) \tilde{r}_{\mu}^{(1)}(\mathrm{V})$ \\
\hline
\end{tabular}

brackets refer to the relative angular momentum between the daughter particles. The amplitudes and the relative phases between the different decay modes are determined with a maximum likelihood fit.

\section{A. Likelihood function construction}

The unbinned maximum likelihood fit is performed by minimizing the negative log likelihood (NLL) of the observed events $\left(N_{\text {data }}\right)$ and the MC-simulated background events $\left(N_{\mathrm{bkg}}\right)$,

$$
\mathrm{NLL}=-\left[\sum_{k}^{N_{\text {data }}} \ln f_{S}\left(p_{j}^{k}\right)+\sum_{k^{\prime}}^{N_{\text {bkg }}} w_{k^{\prime}}^{\mathrm{bkg}} \ln f_{S}\left(p_{j}^{k^{\prime}}\right)\right],
$$

where the indices $k$ and $k^{\prime}$ refer to the $k$ th event of the data sample and the $k^{\prime}$ th background event, respectively. The index $j$ refers to the $j$ th particle in the final state, $f_{S}\left(p_{j}\right)$ is the signal probability density function (PDF) in terms of the final four-momentum $p_{j}$, and $w_{k^{\prime}}^{\mathrm{bkg}}$ is the weight of the $k^{\prime}$ th background event. The contribution from the background is subtracted by assigning a negative weight to the background events.

The signal $\mathrm{PDF} f_{S}\left(p_{j}\right)$ is given by

$$
f_{S}\left(p_{j}\right)=\frac{\epsilon\left(p_{j}\right)\left|M\left(p_{j}\right)\right|^{2} R_{4}\left(p_{j}\right)}{\int \epsilon\left(p_{j}\right)\left|M\left(p_{j}\right)\right|^{2} R_{4}\left(p_{j}\right) d p_{j}},
$$

where $M\left(p_{j}\right)$ is the total decay amplitude describing the dynamics of the $D^{+}$decays, $\epsilon\left(p_{j}\right)$ is the detection efficiency parametrized in terms of the final four-momentum $p_{j} . R_{4}\left(p_{j}\right) d p_{j}$ is the standard element of four-body phase space, which is given by

$$
R_{4}\left(p_{j}\right) d p_{j}=\delta^{4}\left(p_{D^{0}}-\sum_{j}^{4} p_{j}\right) \prod_{j}^{4} \frac{d^{3} \mathbf{p}_{j}}{(2 \pi)^{3} 2 E_{j}} .
$$

The $\epsilon\left(p_{j}\right)$ in the numerator of Eq. (4) is independent of the fitted variables, leading to a constant term in minimizing the likelihood and can be ignored in the fit. The normalization integral of Eq. (4) is performed with a MC technique, which is then given by

$$
\int \epsilon\left(p_{j}\right)\left|M\left(p_{j}\right)\right|^{2} R_{4}\left(p_{j}\right) d p_{j}=\frac{1}{N_{\mathrm{MC}}} \sum_{k_{\mathrm{MC}}}^{N_{\mathrm{MC}}} \frac{\left.\mid M\left(p_{j}^{k_{\mathrm{MC}}}\right)\right)\left.\right|^{2}}{\left|M^{\text {gen }}\left(p_{j}^{k_{\mathrm{MC}}}\right)\right|^{2}},
$$

where $k_{\mathrm{MC}}$ is the index of the $k$ th event of the MC sample and $N_{\mathrm{MC}}$ is the number of the selected MC events. $M^{\text {gen }}\left(p_{j}\right)$ is the PDF function used to generate the MC sample for the integration.

This analysis uses an isobar model formulation in which the total decay amplitude $M\left(p_{j}\right)$ is given by the coherent sum over all contributing amplitudes,

$$
M\left(p_{j}\right)=\sum_{n} \rho_{n} e^{i \phi_{n}} A_{n}\left(p_{j}\right),
$$

where $\rho_{n}$ and $\phi_{n}$ are the magnitude and phase of the $n$th amplitude, respectively. The $n$th amplitude $A_{n}\left(p_{j}\right)$ is given by

$A_{n}\left(p_{j}\right)=P_{n}^{(1)}\left(m_{1}\right) P_{n}^{(2)}\left(m_{2}\right) S_{n}\left(p_{j}\right) B_{n}^{(1)}\left(p_{j}\right) B_{n}^{(2)}\left(p_{j}\right) B_{n}^{(D)}\left(p_{j}\right)$,

where the indices 1 and 2 correspond to the two intermediate resonances. $S_{n}\left(p_{j}\right)$ is the spin factor, $P_{n}^{\alpha}\left(m_{\alpha}\right)$ and $B_{n}^{\alpha}\left(p_{j}\right) \quad(\alpha=1,2)$ are the propagator and the BlattWeisskopf barrier factor [17], respectively, and $B_{n}^{D}\left(p_{j}\right)$ is the Blatt-Weisskopf barrier factor of the $D^{+}$decay. The parameters $m_{1}$ and $m_{2}$ in the propagators are the invariant masses of the corresponding resonances. For nonresonant contributions with orbital angular momentum between the daughters, we set the propagator to unity. This means that the amplitude has negligible $m$ dependence. Since the $D^{+} \rightarrow K_{S}^{0} \pi^{+} \pi^{+} \pi^{-}$decay contains two identical $\pi^{+} \mathrm{s}$ in the final state, $A_{n}\left(p_{j}\right)$ is symmetrized by exchanging the two $\pi^{+} \mathrm{s}$ to take into account the Bose symmetry.

\section{Spin factor}

The spin factor $S_{n}\left(p_{j}\right)$ is constructed with the covariant tensor formalism [5]. The amplitudes with angular momenta larger than 2 are not considered due to the limited phase space. For a specific process $a \rightarrow b c$, the covariant tensors $\tilde{t}_{\mu_{1} \cdots \mu_{l}}^{L}$ for the final states of pure orbital angular momentum $L$ are constructed from the relevant momenta $p_{a}, p_{b}, p_{c}$ [5],

$$
\tilde{t}_{\mu_{1} \cdots \mu_{L}}^{L}=(-1)^{L} P_{\mu_{1} \cdots \mu_{L} \nu_{1} \cdots \nu_{L}}^{(L)} r^{\nu_{1}} \cdots r^{\nu_{L}},
$$

where $r=p_{b}-p_{c} . P_{\mu_{1} \cdots \mu_{L} \nu_{1} \cdots \nu_{L}}^{(L)}$ is the spin projection operator and is defined as

$$
P_{\mu \nu}^{(1)}=-g_{\mu \nu}+\frac{p_{a \mu} p_{a \nu}}{p_{a}^{2}}
$$


for spin 1 , and

$P_{\mu_{1} \mu_{2} \nu_{1} \nu_{2}}^{(2)}=\frac{1}{2}\left(P_{\mu_{1} \nu_{1}}^{(1)} P_{\mu_{2} \nu_{2}}^{(1)}+P_{\mu_{1} \nu_{2}}^{(1)} P_{\mu_{2} \nu_{1}}^{(1)}\right)-\frac{1}{3} P_{\mu_{1} \mu_{2}}^{(1)} P_{\nu_{1} \nu_{2}}^{(1)}$

for spin 2 .

The spin factors of the decay modes used in the analysis are listed in Table I. We use $\tilde{T}_{\mu_{1} \cdots \mu_{L}}^{(L)}$ to represent the decay of the $D^{+}$meson and $\tilde{t}_{\mu_{1} \cdots \mu_{L}}^{(L)}$ to represent the decay of the intermediate state.

\section{Blatt-Weisskopf barrier factors}

For the process $a \rightarrow b c$, the Blatt-Weisskopf barrier factor [17] $B_{L}\left(p_{j}\right)$ is parametrized as a function of the angular momentum $L$ and the momentum $q$ of the daughter $b$ or $c$ in the rest system of $a$,

$$
B_{L}(q)=z^{L} X_{L}(q),
$$

where $z=q R$. $R$ is the effective radius of the barrier, which is fixed to $3.0 \mathrm{GeV}^{-1}$ for the intermediate resonances and $5.0 \mathrm{GeV}^{-1}$ for the $D^{+}$meson. $X_{L}(q)$ is given by

$$
\begin{gathered}
X_{L=0}(q)=1, \\
X_{L=1}(q)=\sqrt{\frac{2}{z^{2}+1}}, \\
X_{L=2}(q)=\sqrt{\frac{13}{z^{4}+3 z^{2}+9}} .
\end{gathered}
$$

With the invariant mass squared $s_{a / b / c}$ of the particle $a / b / c, q$ is

$$
q=\sqrt{\frac{\left(s_{a}+s_{b}-s_{c}\right)^{2}}{4 s_{a}}-s_{b}}
$$

\section{Resonance line shapes}

The propagator $P(m)$ describes the line shape of the intermediate resonance. The resonances $K^{*-}, \bar{K}_{1}(1400)^{0}$, $a_{1}(1260)^{+}$and $\bar{K}(1460)^{0}$ are parametrized with a relativistic Breit-Wigner (RBW) line shape

$$
P^{\mathrm{RBW}}(m)=\frac{1}{m_{0}^{2}-m^{2}-i m_{0} \Gamma(m)},
$$

where $m_{0}$ is the mass of resonance and $\Gamma(m)$ is the massdependent width. The latter is expressed as

$$
\Gamma(m)=\Gamma_{0}\left(\frac{q}{q_{0}}\right)^{2 L+1}\left(\frac{m_{0}}{m}\right)\left(\frac{X_{L}(q)}{X_{L}\left(q_{0}\right)}\right)^{2},
$$

where $\Gamma_{0}$ is the width of resonance and $q_{0}$ denotes the value of $q$ at $m=m_{0}$. The $\omega$ and $K_{1}(1270)^{-}$are parametrized as a RBW with a constant width $\Gamma(m)=\Gamma_{0}$.
The resonance $\rho^{0}$ is described by the Gounaris-Sakurai (GS) function $P_{\rho}^{\mathrm{GS}}(m)$ with the $\rho-\omega$ interference taken into account $[18,19]$,

$$
P_{\rho-\omega}(m)=P_{\rho}^{\mathrm{GS}}(m)\left\{1+\rho_{\omega} e^{i \phi_{\omega}} P_{\omega}^{\mathrm{RBW}}(m)\right\},
$$

where $\rho_{\omega}$ and $\phi_{\omega}$ are the relative magnitude and phase, respectively. $P_{\rho}^{\mathrm{GS}}(m)$ is given by

$$
P_{\rho}^{\mathrm{G} S}(m)=\frac{1+d \frac{\Gamma_{0}}{m_{0}}}{m_{0}^{2}-m^{2}+f(m)-i m_{0} \Gamma(m)},
$$

where

$$
\begin{aligned}
f(m)= & \Gamma_{0} \frac{m_{0}^{2}}{q_{0}^{3}}\left\{q^{2}\left(h(m)-h\left(m_{0}\right)\right)\right. \\
& \left.+\left.\left(m_{0}^{2}-m^{2}\right) q_{0}^{2} \frac{d h}{d\left(m^{2}\right)}\right|_{m^{2}=m_{0}^{2}}\right\},
\end{aligned}
$$

and the function $h(m)$ is defined as

$$
h(m)=\frac{2}{\pi} \frac{q}{m} \ln \left(\frac{m+2 q}{2 m_{\pi}}\right),
$$

with

$\left.\frac{d h}{d\left(m^{2}\right)}\right|_{m^{2}=m_{0}^{2}}=h\left(m_{0}\right)\left[\left(8 q_{0}^{2}\right)^{-1}-\left(2 m_{0}^{2}\right)^{-1}\right]+\left(2 \pi m_{0}^{2}\right)^{-1}$,

where $m_{\pi}$ is the charged pion mass [4]. The normalization condition at $P^{\mathrm{GS}}(0)$ fixes the parameter $d=f(0) /\left(\Gamma_{0} m_{0}\right)$. It is found to be [18]

$$
d=\frac{3}{\pi} \frac{m_{\pi}^{2}}{q_{0}^{2}} \ln \left(\frac{m_{0}+2 q_{0}}{2 m_{\pi}}\right)+\frac{m_{0}}{2 \pi q_{0}}-\frac{m_{\pi}^{2} m_{0}}{\pi q_{0}^{3}} .
$$

The resonance $f_{0}(500)$ is parametrized with the formula given in Ref. [20], which is identical to Eq. (17) with $\Gamma(m)$ being decomposed into two parts,

$$
\Gamma(m)=g_{1} \frac{\rho_{\pi \pi}(m)}{\rho_{\pi \pi}\left(m_{0}\right)}+g_{2} \frac{\rho_{4 \pi}(m)}{\rho_{4 \pi}\left(m_{0}\right)}
$$

and

$$
g_{1}=\left(b_{1}+b_{2} m^{2}\right) \frac{m^{2}-m_{\pi}^{2} / 2}{m_{0}^{2}-m_{\pi}^{2} / 2} e^{\left(m_{0}^{2}-m^{2}\right) / a} .
$$

Here, $\rho_{\pi \pi}$ is the phase space of the $\pi^{+} \pi^{-}$system and $\rho_{4 \pi}$ is the $4 \pi$ phase space approximated by $\sqrt{1-16 m_{\pi}^{2} / m^{2}}$ / $\left[1+e^{3.5\left(2.8-m^{2}\right)}\right][20]$. The parameters $b_{1}, b_{2}$, and $a$ are fixed to the values given in Ref. [21]. 
TABLE II. $\left(K_{S}^{0} \pi^{-}\right)_{S \text {-wave }}$ parameters, obtained from the fit to the $D^{0} \rightarrow K_{S}^{0} \pi^{+} \pi^{-}$Dalitz plot in Ref. [23]. The uncertainties are statistical.

\begin{tabular}{lc}
\hline \hline$M\left(\mathrm{GeV} / c^{2}\right)$ & $1.463 \pm 0.002$ \\
$\Gamma\left(\mathrm{GeV} / c^{2}\right)$ & $0.233 \pm 0.005$ \\
$F$ & $0.80 \pm 0.09$ \\
$\phi_{F}$ & $2.33 \pm 0.13$ \\
$R$ & $1($ fixed $)$ \\
$\phi_{R}$ & $-5.31 \pm 0.04$ \\
$a$ & $1.07 \pm 0.11$ \\
$r$ & $-1.8 \pm 0.3$ \\
\hline \hline
\end{tabular}

The resonance $K_{0}^{*}(1430)^{-}$is considered in a $K \pi S$-wave [denoted as $\left(K_{S}^{0} \pi^{-}\right)_{S \text {-wave }}$ ] parametrization extracted from the scattering data [22]. The same parametrization was used in Ref. [23],

$$
P^{S \text {-wave }}\left(m_{K \pi}\right)=F \sin \delta_{F} e^{i \delta_{F}}+R \sin \delta_{R} e^{i \delta_{R}} e^{i 2 \delta_{F}},
$$

with

$$
\begin{gathered}
\delta_{F}=\phi_{F}+\cot ^{-1}\left[\frac{1}{a q}+\frac{r q}{2}\right], \\
\delta_{R}=\phi_{R}+\tan ^{-1}\left[\frac{M \Gamma\left(m_{K \pi}\right)}{M^{2}-m_{K \pi}^{2}}\right],
\end{gathered}
$$

where $a$ and $r$ denote the scattering length and effective interaction length, respectively. $F\left(\phi_{F}\right)$ and $R\left(\phi_{R}\right)$ are the relative magnitudes (phases) for the nonresonant and resonant terms, and $q$ and $\Gamma\left(m_{K \pi}\right)$ are defined as in Eqs. (16) and (18), respectively. In the fit, the parameters $M, \Gamma, F, \phi_{F}, R, \phi_{R}, a$ and $r$ are fixed to the values obtained from the fit to the $D^{0} \rightarrow K_{S}^{0} \pi^{+} \pi^{-}$Dalitz plot in Ref. [23], given in Table II.

\section{B. Fit fraction}

The fit fraction (FF) for an amplitude or a component (a certain subset of amplitudes) is calculated using a large set of generation-level PHSP MC samples by

$$
\mathrm{FF}(n)=\frac{\sum_{k=1}^{N_{\text {gen }}}\left|\tilde{A}_{\mathbf{n}}\left(p_{j}^{k}\right)\right|^{2}}{\sum_{k=1}^{N_{\text {gen }}}\left|M\left(p_{j}^{k}\right)\right|^{2}},
$$

where $\tilde{A}_{\mathbf{n}}\left(p_{j}^{k}\right)$ is either the $n$th amplitude $\left(\tilde{A}_{\mathbf{n}}\left(p_{j}^{k}\right)=\right.$ $\left.\rho_{n} e^{i \phi_{n}} A_{n}\left(p_{j}^{k}\right)\right)$ or the $\mathbf{n}$ th component of a coherent sum of amplitudes $\left(\tilde{A}_{\mathbf{n}}\left(p_{j}^{k}\right)=\sum \rho_{n_{i}} e^{i \phi_{n_{i}}} A_{n_{i}}\left(p_{j}^{k}\right)\right)$ and $N_{\mathrm{gen}}$ is the number of the PHSP MC events. Note that the sum of the FFs is not necessarily equal to unity due to the interferences among the contributing amplitudes.

To obtain the statistical uncertainties of the FFs, the FFs are calculated 500 times by randomly varying the floated parameters according to the full covariance matrix.
The distribution for each amplitude or each component is fitted with a Gaussian function. The width of the Gaussian function is the statistical uncertainty of the corresponding FF.

\section{RESULTS}

We start the fit of the data by considering the amplitudes containing $K^{*-}, \rho^{0}, \bar{K}_{1}(1270)^{0}, \bar{K}_{1}(1400)^{0}, a_{1}(1260)^{+}$ resonances, as these resonances are clearly observed in the corresponding invariant mass spectra. We then add amplitudes with resonances listed in the PDG [4] and nonresonant components until no additional amplitude has a significance larger than $5 \sigma$. To avoid either artificial or missing components, the total FF of each fit in the procedure is required to be less than 1.5. The cases of high correlation are also avoided, which is discussed in the next paragraph. In addition, in the iteration of adding amplitudes by comparing with the previous step, a better fit quality is required. The statistical significance for any new amplitude is calculated from the change of the log-likelihood value $\Delta$ (NLL) and the change of the degrees of freedom $\Delta \nu$. In the fits, the amplitude and phase of $D^{+} \rightarrow K_{S}^{0} a_{1}(1260)^{+}\left(\rho^{0} \pi^{+}[S]\right)$ are fixed to 1 and 0 as the reference, while the magnitudes and phases of the other amplitudes are floating. Here, $[\mathrm{S}]$ means the angular momentum of the $\rho^{0} \pi^{+}$combination is $0(S$ wave). The corresponding $D$-wave amplitude $D^{+} \rightarrow$ $K_{S}^{0} a_{1}(1260)^{+}\left(\rho^{0} \pi^{+}[D]\right)$ is found to have a FF of about $1 \%$ of the $S$ wave, which is consistent with both BESIII and LHCb amplitude analyses on $D^{0} \rightarrow K^{-} \pi^{+} \pi^{+} \pi^{-}$[24,25]. We consider therefore this $D$-wave amplitude in the nominal fit although its significance is $4.3 \sigma$.

The resonant term $D^{+} \rightarrow K_{S}^{0} a_{1}(1260)^{+}\left(\rho^{0} \pi^{+}[S]\right)$ and its nonresonant partner $D^{+} \rightarrow K_{S}^{0}\left(\rho^{0} \pi^{+}[S]\right)_{A}$ (the subscript $A$ represents the axial-vector nonresonant state for the $\rho^{0} \pi^{+}$ combination) are both found with significances greater than $10 \sigma$, while they are highly correlated because of the same angular distribution and large common region in phase space. For the resonant term in the fit model with the nonresonant partner, its FF becomes highly uncertain and is significantly different to the one in the fit model without the nonresonant partner. However the combined FF of these two amplitudes is almost unchanged. We, therefore, only consider the resonant term. Similar cases are also found with the amplitude pairs of $D^{+} \rightarrow$ $\bar{K}(1460)^{0}\left(K_{S}^{0} \rho^{0}\right) \pi^{+} \quad$ and $\quad D^{+} \rightarrow\left(K_{S}^{0} \rho^{0}\right)_{P} \pi^{+}, \quad D^{+} \rightarrow$ $\bar{K}(1460)^{0}\left(K^{*-} \pi^{+}\right) \pi^{+}$and $D^{+} \rightarrow\left(K^{*-} \pi^{+}\right)_{P} \pi^{+}$, as well as $D^{+} \rightarrow \bar{K}_{1}(1650)^{0}\left(K^{*-} \pi^{+}[S]\right) \pi^{+}$and $D^{+} \rightarrow\left(K^{*-} \pi^{+}[S]\right)_{A} \pi^{+}$. Throughout this paper, we denote $K^{*-} \rightarrow K_{S}^{0} \pi^{-}$and $\rho^{0} \rightarrow \pi^{+} \pi^{-}$, which is also included in the FFs and BFs of corresponding submodes. In the nominal fit, we only use the resonant terms, as done in the analysis of Mark III [26]. 

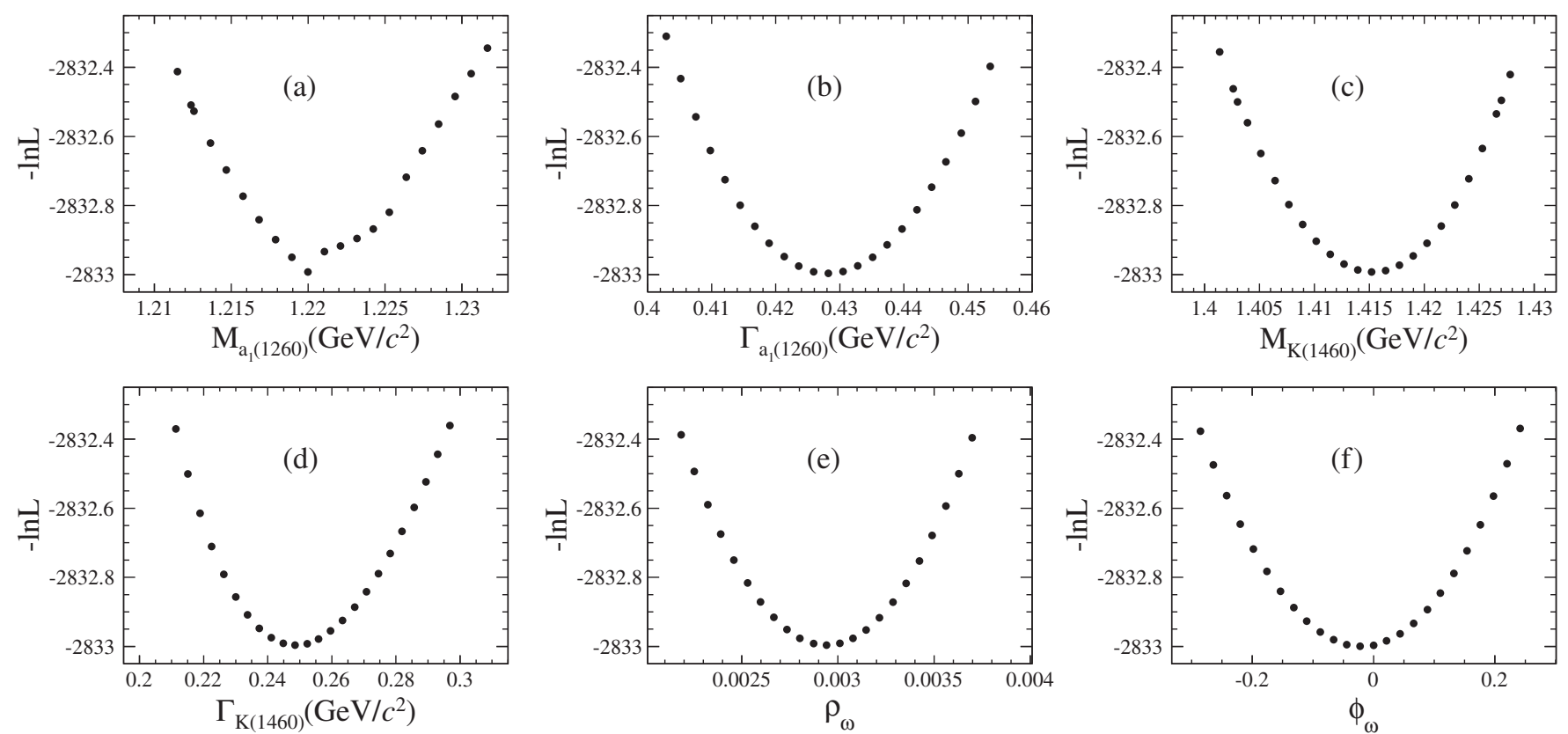

FIG. 2. Likelihood scans of the masses [(a) and (c)] and widths [(b) and (d)] of $a_{1}(1260)^{+}$and $\bar{K}(1460)^{0}$, respectively, as well as the relative magnitude (e) and phase (f) of $\omega$ in the $\rho-\omega$ mixing.

The masses and widths of $\rho^{0}, \omega, K^{*-}, \bar{K}_{1}(1270)^{0}$, $\bar{K}_{1}(1400)^{0}$, and $\bar{K}_{1}(1650)^{0}$ are fixed at the values from the PDG [4]. Since there are no world average values for the masses and widths of $a_{1}(1260)^{+}$and $\bar{K}(1460)^{0}$ and the resonances lie on the upper boundary of the corresponding invariant mass spectrum, their values are determined by likelihood scans. The values of the parameters related to $\rho-\omega$ mixing are also determined by likelihood scans. The scan results are

$$
\begin{aligned}
m_{a_{1}(1260)^{+}} & =1220.0_{-7.6}^{+9.5} \mathrm{MeV} / c^{2}, \\
\Gamma_{a_{1}(1260)^{+}} & =428.2_{-22.2}^{+23.0} \mathrm{MeV} / c^{2}, \\
m_{\bar{K}(1460)^{0}} & =1415.2_{-12.2}^{+11.8} \mathrm{MeV} / c^{2}, \\
\Gamma_{\bar{K}(1460)^{0}} & =248.5_{-33.4}^{+40.8} \mathrm{MeV} / c^{2}, \\
\rho_{\omega} & =(2.94 \pm 0.69) \times 10^{-3}, \\
\phi_{\omega} & =-0.02 \pm 0.23,
\end{aligned}
$$

where the uncertainties are statistical only. In the nominal fit, these parameters are set to be the values determined by likelihood scans. The scan results are shown in Fig. 2.

In Fig. 2(a), three scan points at the right of the minimum point are higher than smooth scan expectations due to the correlation between the states with resonances $a_{1}(1260)^{+}$ or $\bar{K}(1460)^{0}$ involved.

Finally, our nominal fit model includes 13 amplitudes (labeled as I, II, III, ..., XIII), in which eight of them can be summarized into four different components. To quantify the fit quality for this unbinned likelihood fit, an unbinned "mixed-sample method" is performed, which is described in Refs. [27,28]. With this method, the p-value is $25.5 \%$. The projections of the invariant mass spectra and the distribution of $\chi$ are shown in Fig. 3. All the amplitudes and the corresponding significances and phases, as well as the FFs of amplitudes and components are listed in Table III, where the last row of each box is the coherent sum of the preceding amplitudes (components). For the phases and FFs, the first and second uncertainties are statistical and systematic, respectively. The systematic uncertainties are discussed below. Other tested amplitudes when determining the nominal fit model, but finally not used, are listed in Appendix A. The interference fit fractions between each amplitude are given in Appendix B.

\section{SYSTEMATIC UNCERTAINTIES}

The systematic uncertainties are categorized into the following sources: (I) masses and widths of the intermediate resonances, (II) effective radius of intermediate resonances and $D^{+}$, (III) parameters in $K_{S}^{0} \pi^{+} S$-wave parametrization, (IV) parameters in $\rho-\omega$ mixing parametrization, (V) line shape of $f_{0}(500)$, (VI) line shape of $a_{1}(1260)$, (VII) effect from peaking background, (VIII) effect from general background, and (IX) fit procedure. The systematic uncertainties of the phases of amplitudes and the FFs of amplitudes and components due to different contributions are given in Tables IV and V, respectively. These uncertainties are given in units of standard deviations $\sigma_{\text {stat }}$ and are added in quadrature to obtain the total systematic uncertainties, as they are uncorrelated. 

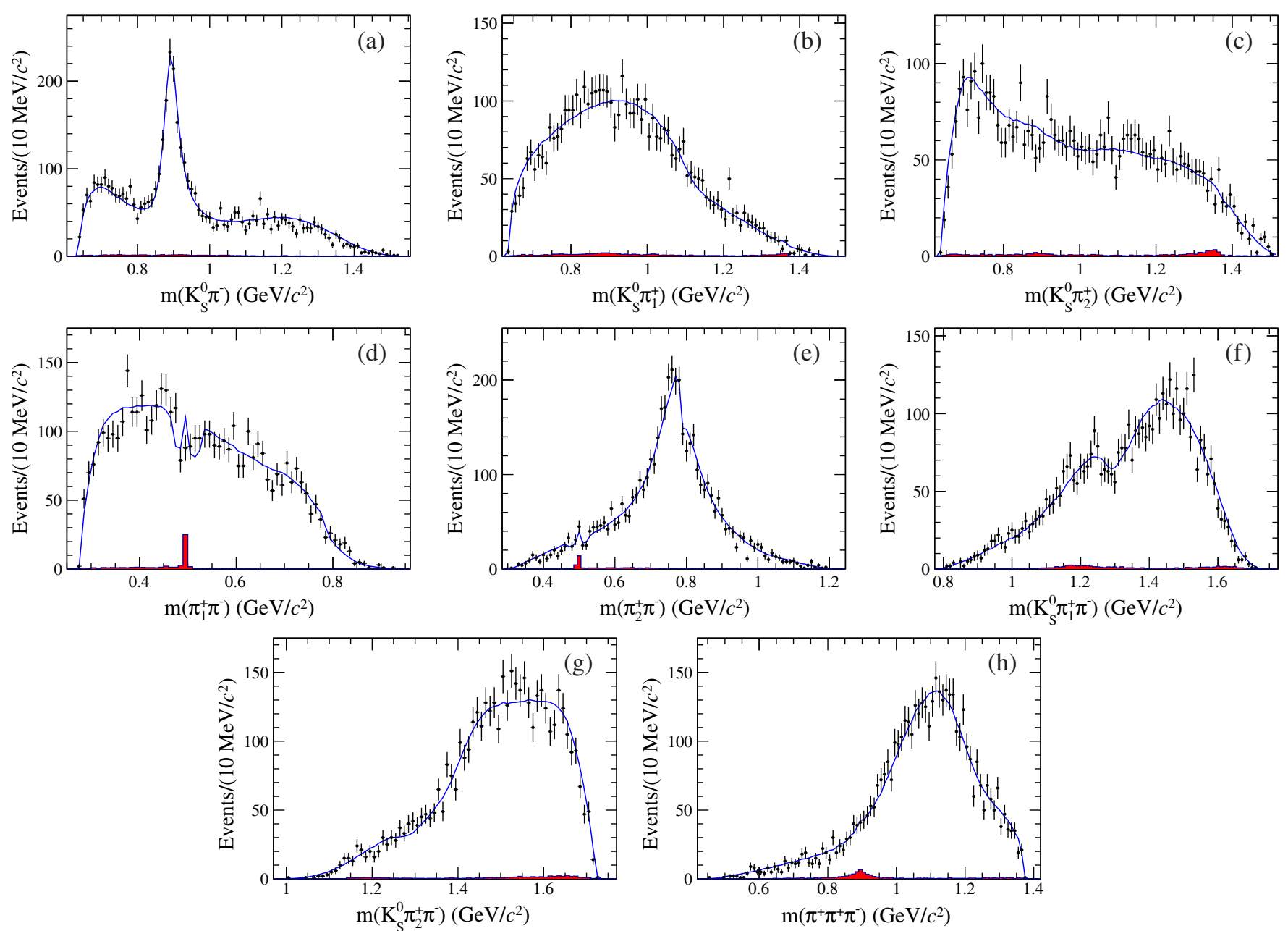

FIG. 3. The projections of (a) $K_{S}^{0} \pi^{-}$, (b) $K_{S}^{0} \pi_{1}^{+}$, (c) $K_{S}^{0} \pi_{2}^{+}$, (d) $\pi_{1}^{+} \pi^{-}$, (e) $\pi_{2}^{+} \pi^{-}$, (f) $K_{S}^{0} \pi_{1}^{+} \pi^{-}$, (g) $K_{S}^{0} \pi_{2}^{+} \pi^{-}$, and (h) $\pi^{+} \pi^{+} \pi^{-}$invariant mass spectra, where the dots with error are data, and the curves are the fit projections. The small red histogram in each projection shows the $D^{+} \rightarrow K_{S}^{0} K_{S}^{0} \pi^{+}$peaking background. The dip around the $K_{S}^{0}$ peak comes from the used requirement to suppress the $D^{+} \rightarrow K_{S}^{0} K_{S}^{0} \pi^{+}$peaking background. For the identical pions, the one resulting in a lower $\pi^{+} \pi^{-}$invariant mass is denoted as $\pi_{1}^{+}$; the other is denoted as $\pi_{2}^{+}$.

To estimate the systematic uncertainties, the fit is altered to investigate the effect from each source. For the masses and widths of the intermediate resonances given by the PDG [4], they are shifted within the uncertainties from the PDG [4]. The masses and widths of $a_{1}(1260)^{+}$and $\bar{K}(1460)$, as well as the relative magnitude and phase of $\omega$ in $\rho-\omega$ parametrization are shifted within the uncertainties given by the likelihood scans. The barrier effective radius $\mathrm{R}$ is varied within $\pm 1 \mathrm{GeV}^{-1}$. The input parameters of $K_{S}^{0} \pi^{+} S$-wave model are varied within their uncertainties given by Ref. [23]. For the resonance $f_{0}(500)$, the propagator is replaced by the RBW function with mass and width fixed at $526 \mathrm{MeV} / c^{2}$ and $535 \mathrm{MeV}$ [21], respectively. For the resonance $a_{1}(1260)^{+}$, a constant width Breit-Wigner with mass and width determined by the fit is used to estimate the effect from the $a_{1}(1260)^{+}$line shape. For the effects from different line shapes, only the changes in the fit fractions are given. Since different propagators have different normalization factors, for the amplitude with $f_{0}(500)$ involved, the shift effects on the FF are only considered. The effect from the peaking background $D^{+} \rightarrow K_{S}^{0} K_{S}^{0} \pi^{+}$is estimated by altering the number of background events to be half of that in the nominal fit. The uncertainty from general background is studied by taking the background events into account, which are estimated from the average $M_{\mathrm{BC}}$ $\left(\left(M_{\mathrm{BC}}\left(D_{\text {tag }}\right)+M_{\mathrm{BC}}\left(D_{\text {signal }}\right)\right) / 2\right)$ sideband region of $[1.830,1.858] \mathrm{GeV} / c^{2}$. Individual changes of the results with respect to the nominal one are taken as the corresponding systematic uncertainties.

To evaluate the uncertainty from the fit procedure, we generate 300 sets of signal MC samples according to the nominal results in this analysis. Each sample, which has equivalent size as the data, is analyzed with the same 
TABLE III. Significances and phases for different amplitudes, labeled as I, II, III, .., XIII, respectively, as well as FFs for amplitudes and components (the last row of each box), where the first and second uncertainties are statistical and systematic, respectively. The $f_{0}(500)$ and $\rho^{0}$ resonances decay to $\pi^{+} \pi^{-}$, and the $K^{*-}$ resonance decays to $K_{S}^{0} \pi^{-}$.

\begin{tabular}{|c|c|c|c|c|}
\hline & Amplitude & Significance $(\sigma)$ & Phase & $\mathrm{FF}$ \\
\hline I & $D^{+} \rightarrow K_{S}^{0} a_{1}(1260)^{+}\left(\rho^{0} \pi^{+}[S]\right)$ & $>10$ & 0.0 (fixed) & $0.384 \pm 0.021 \pm 0.041$ \\
\hline II & $\begin{array}{l}D^{+} \rightarrow K_{S}^{0} a_{1}(1260)^{+}\left(\rho^{0} \pi^{+}[D]\right) \\
D^{+} \rightarrow K_{S}^{0} a_{1}(1260)^{+}\left(\rho^{0} \pi^{+}\right)\end{array}$ & $\begin{array}{l}4.3 \\
\cdots\end{array}$ & $\begin{array}{c}-1.55 \pm 0.16 \pm 0.22 \\
\ldots\end{array}$ & $\begin{array}{l}0.004 \pm 0.002 \pm 0.001 \\
0.403 \pm 0.021 \pm 0.041\end{array}$ \\
\hline III & $D^{+} \rightarrow K_{S}^{0} a_{1}(1260)^{+}\left(f_{0}(500) \pi^{+}\right)$ & $>10$ & $-1.82 \pm 0.08 \pm 0.10$ & $0.055 \pm 0.007 \pm 0.018$ \\
\hline IV & $D^{+} \rightarrow \bar{K}_{1}(1400)^{0}\left(K^{*-} \pi^{+}[S]\right) \pi^{+}$ & $>10$ & $-2.68 \pm 0.05 \pm 0.07$ & $0.221 \pm 0.012 \pm 0.016$ \\
\hline $\mathrm{V}$ & $\begin{array}{l}D^{+} \rightarrow \bar{K}_{1}(1400)^{0}\left(K^{*-} \pi^{+}[D]\right) \pi^{+} \\
D^{+} \rightarrow \bar{K}_{1}(1400)^{0}\left(K^{*-} \pi^{+}\right) \pi^{+}\end{array}$ & $\begin{array}{l}>10 \\
\cdots\end{array}$ & $\begin{array}{c}-2.24 \pm 0.10 \pm 0.07 \\
\cdots\end{array}$ & $\begin{array}{l}0.015 \pm 0.002 \pm 0.001 \\
0.216 \pm 0.012 \pm 0.011\end{array}$ \\
\hline VI & $D^{+} \rightarrow \bar{K}_{1}(1270)^{0}\left(K_{S}^{0} \rho^{0}[S]\right) \pi^{+}$ & 9.7 & $-0.56 \pm 0.09 \pm 0.11$ & $0.024 \pm 0.003 \pm 0.006$ \\
\hline XIII & $D^{+} \rightarrow \bar{K}(1460)^{0}\left(K^{*-} \pi^{+}\right) \pi^{+}$ & $>10$ & $-2.50 \pm 0.07 \pm 0.06$ & $0.068 \pm 0.006 \pm 0.010$ \\
\hline IX & $D^{+} \rightarrow \bar{K}(1460)^{0}\left(K_{S}^{0} \rho^{0}\right) \pi^{+}$ & 6.1 & $-2.65 \pm 0.18 \pm 0.25$ & $0.008 \pm 0.002 \pm 0.005$ \\
\hline $\mathrm{X}$ & $D^{+} \rightarrow \bar{K}_{1}(1650)^{0}\left(K^{*-} \pi^{+}[S]\right) \pi^{+}$ & 6.5 & $-0.95 \pm 0.14 \pm 0.22$ & $0.016 \pm 0.004 \pm 0.014$ \\
\hline VII & $D^{+} \rightarrow\left(K_{S}^{0} \rho^{0}[S]\right)_{A} \pi^{+}$ & $>10$ & $-1.88 \pm 0.08 \pm 0.05$ & $0.057 \pm 0.007 \pm 0.023$ \\
\hline VIII & $\begin{array}{l}D^{+} \rightarrow\left(K_{S}^{0} \rho^{0}[D]\right)_{A} \pi^{+} \\
D^{+} \rightarrow\left(K_{S}^{0} \rho^{0}\right)_{A} \pi^{+}\end{array}$ & $\begin{array}{l}7.0 \\
\cdots\end{array}$ & $\begin{array}{c}2.77 \pm 0.12 \pm 0.14 \\
\ldots\end{array}$ & $\begin{array}{l}0.008 \pm 0.002 \pm 0.003 \\
0.064 \pm 0.007 \pm 0.034\end{array}$ \\
\hline XI & $D^{+} \rightarrow\left(K_{S}^{0}\left(\pi^{+} \pi^{-}\right)_{S}\right)_{A} \pi^{+}$ & $>10$ & $-3.08 \pm 0.06 \pm 0.04$ & $0.064 \pm 0.005 \pm 0.007$ \\
\hline XII & $\begin{array}{l}D^{+} \rightarrow\left(\left(K_{S}^{0} \pi^{+}\right)_{S \text {-wave }} \pi^{-}\right)_{P} \pi^{+} \\
D^{+} \rightarrow K_{S}^{0} \pi^{+} \pi^{+} \pi^{-} \text {nonresonance }\end{array}$ & $\begin{array}{l}>10 \\
\cdots\end{array}$ & $\begin{array}{c}2.10 \pm 0.08 \pm 0.28 \\
\cdots\end{array}$ & $\begin{array}{l}0.017 \pm 0.003 \pm 0.005 \\
0.081 \pm 0.006 \pm 0.009\end{array}$ \\
\hline
\end{tabular}

method as data analysis. We fit the resulting pull distributions, $\frac{V_{\text {input }}-V_{\text {fit }}}{\sigma_{\text {fit }}}$, where $V_{\text {input }}$ is the input value in the generator, and $V_{\text {fit }}$ and $\sigma_{\text {fit }}$ are the output value and the corresponding statistical uncertainty, respectively. Fits to the pull distributions with Gaussian functions show no obvious biases and under- or overestimations on statistical uncertainties. We add in quadrature the mean and the mean error of the pull and multiply this number with the statistical error to get the systematic error. The results are given in Table VI, in which the corresponding uncertainties are the statistical uncertainties of the respective fits.

The effects from tracking/PID efficiency and the kinematic fit arising due to the imperfect modeling of the data by the simulation, as well as the resolution, are also

TABLE IV. Systematic uncertainties of phases for amplitudes. The different sources include (I) masses and widths of the intermediate resonances, (II) effective radius of intermediate resonances and $D^{+}$, (III) parameters in the $K_{S}^{0} \pi^{+} S$-wave parametrization, (IV) parameters in the $\rho-\omega$ mixing parametrization, (V) line shape of the $f_{0}(500)$, (VII) effect from peaking background, (VIII) effect from general background, and (IX) fit procedure.

\begin{tabular}{|c|c|c|c|c|c|c|c|c|c|}
\hline \multirow[b]{2}{*}{ Amplitude } & \multicolumn{8}{|c|}{ Source $\left(\sigma_{\text {stat }}\right)$} & \multirow[b]{2}{*}{ total } \\
\hline & I & II & III & IV & V & VII & VIII & IX & \\
\hline$D^{+} \rightarrow K_{S}^{0} a_{1}(1260)^{+}\left(\rho^{0} \pi^{+}[D]\right)$ & 0.317 & 0.413 & 1.221 & 0.059 & 0.273 & 0.042 & 0.057 & 0.061 & 1.412 \\
\hline$D^{+} \rightarrow K_{S}^{0} a_{1}(1260)^{+}\left(f_{0}(500) \pi^{+}\right)$ & 0.265 & 0.343 & 1.110 & 0.262 & $\cdots$ & 0.220 & 0.058 & 0.071 & 1.243 \\
\hline$D^{+} \rightarrow \bar{K}_{1}(1400)^{0} \pi^{+}\left(K^{*-} \pi^{+}[S]\right)$ & 0.872 & 0.362 & 1.006 & 0.131 & 0.257 & 0.003 & 0.051 & 0.058 & 1.412 \\
\hline$D^{+} \rightarrow \bar{K}_{1}(1400)^{0} \pi^{+}\left(K^{*-} \pi^{+}[D]\right)$ & 0.393 & 0.252 & 0.451 & 0.068 & 0.062 & 0.001 & 0.097 & 0.149 & 0.679 \\
\hline$D^{+} \rightarrow \bar{K}_{1}(1270)^{0} \pi^{+}\left(K_{S}^{0} \rho^{0}[S]\right)$ & 1.135 & 0.349 & 0.123 & 0.021 & 0.012 & 0.131 & 0.121 & 0.121 & 1.213 \\
\hline$D^{+} \rightarrow \bar{K}(1460)^{0}\left(K^{*-} \pi^{+}\right) \pi^{+}$ & 0.786 & 0.032 & 0.152 & 0.049 & 0.128 & 0.028 & 0.092 & 0.054 & 0.820 \\
\hline$D^{+} \rightarrow \bar{K}(1460)^{0}\left(K_{S}^{0} \rho^{0}\right) \pi^{+}$ & 0.573 & 0.022 & 1.249 & 0.023 & 0.261 & 0.070 & 0.062 & 0.139 & 1.409 \\
\hline$D^{+} \rightarrow \bar{K}_{1}(1650)^{0} \pi^{+}\left(K^{*-} \pi^{+}[S]\right)$ & 1.171 & 0.166 & 0.948 & 0.026 & 0.089 & 0.066 & 0.118 & 0.051 & 1.526 \\
\hline$D^{+} \rightarrow\left(K_{S}^{0} \rho^{0}[S]\right)_{A} \pi^{+}$ & 0.539 & 0.307 & 0.217 & 0.015 & 0.061 & 0.007 & 0.115 & 0.050 & 0.672 \\
\hline$D^{+} \rightarrow\left(K_{S}^{0} \rho^{0}[D]\right)_{A} \pi^{+}$ & 0.173 & 0.278 & 1.057 & 0.038 & 0.273 & 0.045 & 0.057 & 0.100 & 1.147 \\
\hline$D^{+} \rightarrow\left(K_{S}^{0}\left(\pi^{+} \pi^{-}\right)_{S}\right)_{A} \pi^{+}$ & 0.254 & 0.508 & 0.442 & 0.072 & 0.010 & 0.058 & 0.092 & 0.050 & 0.733 \\
\hline$D^{+} \rightarrow\left(\left(K_{S}^{0} \pi^{+}\right)_{S \text {-wave }} \pi^{-}\right)_{P} \pi^{+}$ & 0.142 & 0.226 & 3.309 & 0.083 & 0.192 & 0.027 & 0.059 & 0.125 & 3.330 \\
\hline
\end{tabular}


TABLE V. Systematic uncertainties of FFs for amplitudes and components. The different sources include (I) masses and widths of the intermediate resonances, (II) effective radius of intermediate resonances and $D^{+}$, (III) parameters in the $K_{S}^{0} \pi^{+} S$-wave parametrization, (IV) parameters in $\rho-\omega$ mixing parametrization, (V) line shape of the $f_{0}(500)$, (VI) line shape of the $a_{1}(1260)$ (VII) effect from peaking background, (VIII) effect from general background, and (IX) fit procedure.

\begin{tabular}{|c|c|c|c|c|c|c|c|c|c|c|}
\hline \multirow[b]{2}{*}{ Amplitude and component } & \multicolumn{10}{|c|}{ Source $\left(\sigma_{\text {stat }}\right)$} \\
\hline & I & II & III & IV & $\mathrm{V}$ & VI & VII & VIII & IX & total \\
\hline$D^{+} \rightarrow K_{S}^{0} a_{1}(1260)^{+}\left(\rho^{0} \pi^{+}[S]\right)$ & 0.299 & 0.831 & 0.496 & 0.069 & 0.877 & 1.419 & 0.215 & 0.023 & 0.143 & 1.970 \\
\hline$D^{+} \rightarrow K_{S}^{0} a_{1}(1260)^{+}\left(\rho^{0} \pi^{+}[D]\right)$ & 0.137 & 0.335 & 0.032 & 0.078 & 0.014 & 0.367 & 0.028 & 0.054 & 0.085 & 0.533 \\
\hline$D^{+} \rightarrow K_{S}^{0} a_{1}(1260)^{+}\left(\rho^{0} \pi^{+}\right)$ & 0.301 & 0.885 & 0.529 & 0.054 & 0.870 & 1.333 & 0.217 & 0.014 & 0.125 & 1.937 \\
\hline$D^{+} \rightarrow K_{S}^{0} a_{1}(1260)^{+}\left(f_{0}(500) \pi^{+}\right)$ & 0.534 & 0.538 & 2.369 & 0.050 & 0.553 & 0.775 & 0.215 & 0.097 & 0.085 & 2.532 \\
\hline$D^{+} \rightarrow \bar{K}_{1}(1400)^{0} \pi^{+}\left(K^{*-} \pi^{+}[S]\right)$ & 1.260 & 0.094 & 0.306 & 0.003 & 0.093 & 0.098 & 0.177 & 0.174 & 0.060 & 1.332 \\
\hline$D^{+} \rightarrow \bar{K}_{1}(1400)^{0} \pi^{+}\left(K^{*-} \pi^{+}[D]\right)$ & 0.286 & 0.099 & 0.216 & 0.007 & 0.041 & 0.289 & 0.027 & 0.042 & 0.078 & 0.482 \\
\hline$D^{+} \rightarrow \bar{K}_{1}(1400)^{0} \pi^{+}\left(K^{*-} \pi^{+}\right)$ & 0.857 & 0.078 & 0.221 & 0.002 & 0.066 & 0.080 & 0.123 & 0.119 & 0.063 & 0.914 \\
\hline$D^{+} \rightarrow \bar{K}_{1}(1270)^{0} \pi^{+}\left(K_{S}^{0} \rho^{0}[S]\right)$ & 1.151 & 0.274 & 1.511 & 0.071 & 0.480 & 0.633 & 0.172 & 0.061 & 0.086 & 2.088 \\
\hline$D^{+} \rightarrow \bar{K}(1460)^{0}\left(K^{*-} \pi^{+}\right) \pi^{+}$ & 0.288 & 0.081 & 0.162 & 0.001 & 0.048 & 1.687 & 0.016 & 0.016 & 0.071 & 1.723 \\
\hline$D^{+} \rightarrow \bar{K}(1460)^{0}\left(K_{S}^{0} \rho^{0}\right) \pi^{+}$ & 0.365 & 0.546 & 2.288 & 0.044 & 0.374 & 0.347 & 0.194 & 0.153 & 0.058 & 2.448 \\
\hline$D^{+} \rightarrow \bar{K}_{1}(1650)^{0} \pi^{+}\left(K^{*-} \pi^{+}[S]\right)$ & 1.836 & 0.862 & 0.077 & 0.007 & 0.164 & 2.831 & 0.095 & 0.195 & 0.063 & 3.495 \\
\hline$D^{+} \rightarrow\left(K_{S}^{0} \rho^{0}[S]\right)_{A} \pi^{+}$ & 0.644 & 0.758 & 3.139 & 0.036 & 0.124 & 0.027 & 0.154 & 0.037 & 0.058 & 3.300 \\
\hline$D^{+} \rightarrow\left(K_{S}^{0} \rho^{0}[D]\right)_{A} \pi^{+}$ & 0.188 & 0.248 & 0.334 & 0.044 & 0.010 & 1.208 & 0.072 & 0.001 & 0.092 & 1.298 \\
\hline$D^{+} \rightarrow\left(K_{S}^{0} \rho^{0}\right)_{A} \pi^{+}$ & 0.863 & 0.876 & 4.287 & 0.031 & 0.131 & 1.992 & 0.236 & 0.066 & 0.078 & 4.893 \\
\hline$D^{+} \rightarrow\left(K_{S}^{0}\left(\pi^{+} \pi^{-}\right)_{S}\right)_{A} \pi^{+}$ & 0.751 & 0.318 & 0.933 & 0.035 & 0.243 & 0.005 & 0.548 & 0.363 & 0.149 & 1.432 \\
\hline$D^{+} \rightarrow\left(\left(K_{S}^{0} \pi^{+}\right)_{S \text {-wave }} \pi^{-}\right)_{P} \pi^{+}$ & 0.347 & 0.073 & 1.422 & 0.014 & 0.107 & 0.128 & 0.259 & 0.039 & 0.086 & 1.502 \\
\hline$D^{+} \rightarrow K_{S}^{0} \pi^{+} \pi^{+} \pi^{-}$nonresonance & 0.604 & 0.256 & 0.191 & 0.025 & 0.153 & 1.038 & 0.580 & 0.327 & 0.078 & 1.420 \\
\hline
\end{tabular}

TABLE VI. Mean and width of the pull distributions for phases and FFs with statistical uncertainties.

\begin{tabular}{|c|c|c|c|c|}
\hline \multirow[b]{2}{*}{ Amplitude and component } & \multicolumn{2}{|c|}{ Phase } & \multicolumn{2}{|c|}{ FF } \\
\hline & Mean & Width & Mean & Width \\
\hline$D^{+} \rightarrow K_{S}^{0} a_{1}(1260)^{+}\left(\rho^{0} \pi^{+}[S]\right)$ & $\cdots$ & $\cdots$ & $-0.13 \pm 0.06$ & $0.96 \pm 0.04$ \\
\hline$D^{+} \rightarrow K_{S}^{0} a_{1}(1260)^{+}\left(\rho^{0} \pi^{+}[D]\right)$ & $0.01 \pm 0.06$ & $1.01 \pm 0.04$ & $0.06 \pm 0.06$ & $0.96 \pm 0.04$ \\
\hline$D^{+} \rightarrow K_{S}^{0} a_{1}(1260)^{+}\left(\rho^{0} \pi^{+}\right)$ & $\ldots$ & $\cdots$ & $-0.11 \pm 0.06$ & $0.97 \pm 0.04$ \\
\hline$D^{+} \rightarrow K_{S}^{0} a_{1}(1260)^{+}\left(f_{0}(500) \pi^{+}\right)$ & $0.05 \pm 0.05$ & $0.89 \pm 0.04$ & $0.06 \pm 0.06$ & $1.01 \pm 0.04$ \\
\hline$D^{+} \rightarrow \bar{K}_{1}(1400)^{0} \pi^{+}\left(K^{*-} \pi^{+}[S]\right)$ & $-0.03 \pm 0.05$ & $0.92 \pm 0.04$ & $0.00 \pm 0.06$ & $1.03 \pm 0.04$ \\
\hline$D^{+} \rightarrow \bar{K}_{1}(1400)^{0} \pi^{+}\left(K^{*-} \pi^{+}[D]\right)$ & $0.14 \pm 0.05$ & $0.93 \pm 0.04$ & $0.05 \pm 0.06$ & $0.97 \pm 0.04$ \\
\hline$D^{+} \rightarrow \bar{K}_{1}(1400)^{0} \pi^{+}\left(K^{*-} \pi^{+}\right)$ & $\cdots$ & $\cdots$ & $0.02 \pm 0.06$ & $0.97 \pm 0.04$ \\
\hline$D^{+} \rightarrow \bar{K}_{1}(1270)^{0} \pi^{+}\left(K_{S}^{0} \rho^{0}[S]\right)$ & $0.11 \pm 0.05$ & $0.95 \pm 0.04$ & $-0.07 \pm 0.05$ & $0.95 \pm 0.04$ \\
\hline$D^{+} \rightarrow \bar{K}(1460)^{0}\left(K^{*-} \pi^{+}\right) \pi^{+}$ & $-0.02 \pm 0.05$ & $0.91 \pm 0.04$ & $0.05 \pm 0.05$ & $0.95 \pm 0.04$ \\
\hline$D^{+} \rightarrow \bar{K}(1460)^{0}\left(K_{S}^{0} \rho^{0}\right) \pi^{+}$ & $0.13 \pm 0.05$ & $0.94 \pm 0.04$ & $0.03 \pm 0.05$ & $0.95 \pm 0.04$ \\
\hline$D^{+} \rightarrow \bar{K}_{1}(1650)^{0} \pi^{+}\left(K^{*-} \pi^{+}[S]\right)$ & $0.01 \pm 0.05$ & $0.93 \pm 0.04$ & $-0.02 \pm 0.06$ & $1.01 \pm 0.04$ \\
\hline$D^{+} \rightarrow\left(K_{S}^{0} \rho^{0}[S]\right)_{A} \pi^{+}$ & $0.00 \pm 0.05$ & $0.93 \pm 0.04$ & $-0.03 \pm 0.05$ & $0.89 \pm 0.04$ \\
\hline$D^{+} \rightarrow\left(K_{S}^{0} \rho^{0}[D]\right)_{A} \pi^{+}$ & $-0.08 \pm 0.06$ & $1.06 \pm 0.04$ & $0.07 \pm 0.06$ & $1.06 \pm 0.04$ \\
\hline$D^{+} \rightarrow\left(K_{S}^{0} \rho^{0}\right)_{A} \pi^{+}$ & $\cdots$ & $\cdots$ & $0.06 \pm 0.05$ & $0.93 \pm 0.04$ \\
\hline$D^{+} \rightarrow\left(K_{S}^{0}\left(\pi^{+} \pi^{-}\right)_{S}\right)_{A} \pi^{+}$ & $0.00 \pm 0.05$ & $0.87 \pm 0.04$ & $-0.14 \pm 0.05$ & $0.92 \pm 0.04$ \\
\hline$D^{+} \rightarrow\left(\left(K_{S}^{0} \pi^{+}\right)_{S \text {-wave }} \pi^{-}\right)_{P} \pi^{+}$ & $0.11 \pm 0.06$ & $0.97 \pm 0.04$ & $0.07 \pm 0.05$ & $0.93 \pm 0.04$ \\
\hline$D^{+} \rightarrow K_{S}^{0} \pi^{+} \pi^{+} \pi^{-}$nonresonance & $\cdots$ & $\ldots$ & $-0.06 \pm 0.05$ & $0.95 \pm 0.04$ \\
\hline
\end{tabular}


investigated. For tracking/PID efficiency and kinematic fit, a factor related to the correction is considered when calculating the normalization integral of Eq. (4). The difference between the alternative fit and the nominal fit is found to be negligible. The effect from the resolution is estimated from the difference of the pull distribution obtained from these 300 sets of signal MC samples using the generated and reconstructed four-momenta, which is also found to be negligible.

\section{CONCLUSION}

We have determined the intermediate state contributions to the decay $D^{+} \rightarrow K_{S}^{0} \pi^{+} \pi^{+} \pi^{-}$from an amplitude analysis. With the fit fraction of the $n$th component $\operatorname{FF}(n)$ obtained from this analysis, we calculate the corresponding BF: $\mathcal{B}(n)=\mathcal{B}\left(D^{+} \rightarrow K_{S}^{0} \pi^{+} \pi^{+} \pi^{-}\right) \times \mathrm{FF}(n)$, where $\mathcal{B}\left(D^{+} \rightarrow\right.$ $\left.K_{S}^{0} \pi^{+} \pi^{+} \pi^{-}\right)=(2.97 \pm 0.11) \%$ is the total inclusive BF quoted from the PDG [4]. The results on the BFs are shown in Table VII.

Compared with the previous measurements [26], the precisions of the subdecay modes are significantly improved. The dominant intermediate process is $D^{+} \rightarrow$ $K_{S}^{0} a_{1}(1260)^{+}\left(\rho^{0} \pi^{+}\right)$, which agrees with the measurement of Mark III [26]. We also extract the BFs of $D^{+} \rightarrow$ $K_{S}^{0} a_{1}(1260)^{+}\left(f_{0}(500) \pi^{+}\right), D^{+} \rightarrow \bar{K}_{1}(1400)^{0}\left(K^{*-} \pi^{+}\right) \pi^{+}$, and $D^{+} \rightarrow \bar{K}_{1}(1270)^{0}\left(K_{S}^{0} \rho^{0}\right) \pi^{+}$decays for the first time. Comparing with the decay of $D^{0} \rightarrow K^{-} \pi^{+} \pi^{+} \pi^{-}$[24,25], the decay mode $D \rightarrow K a_{1}(1260)$ is found to be the dominant substructure in both $D^{0}$ and $D^{+}$decays. For the two $K_{1}$ states, the contributions from $D \rightarrow K_{1}(1270) \pi$ are at the same level for both $D^{+}$and $D^{0}$ decays. For $D \rightarrow K_{1}(1400) \pi$, the related $\mathrm{BF}$ in $D^{+}$decays is found to be greater than that in $D^{0}$ decay by 1 order of magnitude. These results provide criteria to further investigate the mixture between these two axial-vector kaon states [1-3].

TABLE VII. The results of BFs for different components. The first, second and third errors are statistical, systematical and the uncertainty related to $\mathcal{B}\left(D^{+} \rightarrow K_{S}^{0} \pi^{+} \pi^{+} \pi^{-}\right)$[4], respectively. The $f_{0}(500)$ and $\rho^{0}$ resonances decay to $\pi^{+} \pi^{-}$, and the $K^{*-}$ resonance decays to $K_{S}^{0} \pi^{-}$.

\begin{tabular}{lc}
\hline \hline Component & Branching fraction (\%) \\
\hline$D^{+} \rightarrow K_{S}^{0} a_{1}(1260)^{+}\left(\rho^{0} \pi^{+}\right)$ & $1.197 \pm 0.062 \pm 0.120 \pm 0.044$ \\
$D^{+} \rightarrow K_{S}^{0} a_{1}(1260)^{+}\left(f_{0}(500) \pi^{+}\right)$ & $0.163 \pm 0.021 \pm 0.053 \pm 0.006$ \\
$D^{+} \rightarrow \bar{K}_{1}(1400)^{0}\left(K^{*-} \pi^{+}\right) \pi^{+}$ & $0.642 \pm 0.036 \pm 0.033 \pm 0.024$ \\
$D^{+} \rightarrow \bar{K}_{1}(1270)^{0}\left(K_{S}^{0} \rho^{0}\right) \pi^{+}$ & $0.071 \pm 0.009 \pm 0.019 \pm 0.003$ \\
$D^{+} \rightarrow \bar{K}(1460)^{0}\left(K^{*-} \pi^{+}\right) \pi^{+}$ & $0.202 \pm 0.018 \pm 0.031 \pm 0.007$ \\
$D^{+} \rightarrow \bar{K}(1460)^{0}\left(K_{S}^{0} \rho^{0}\right) \pi^{+}$ & $0.024 \pm 0.006 \pm 0.015 \pm 0.009$ \\
$D^{+} \rightarrow \bar{K}_{1}(1650)^{0}\left(K^{*-} \pi^{+}\right) \pi^{+}$ & $0.048 \pm 0.012 \pm 0.042 \pm 0.002$ \\
$D^{+} \rightarrow K_{S}^{0} \pi^{+} \rho^{0}$ & $0.190 \pm 0.021 \pm 0.103 \pm 0.007$ \\
$D^{+} \rightarrow K_{S}^{0} \pi^{+} \pi^{+} \pi^{-}$ & $0.241 \pm 0.018 \pm 0.026 \pm 0.009$ \\
\hline \hline
\end{tabular}

\section{ACKNOWLEDGMENTS}

The BESIII collaboration thanks the staff of BEPCII and the IHEP computing center for their strong support. This work is supported in part by National Key Basic Research Program of China under Contract No. 2015CB856700; National Natural Science Foundation of China (NSFC) under Contracts No. 11075174, No. 11121092, No. 11425524, No. 11475185, No. 11625523, No. 11635010, and No. 11735014; the Chinese Academy of Sciences (CAS) Large-Scale Scientific Facility Program; the CAS Center for Excellence in Particle Physics (CCEPP); Joint Large-Scale Scientific Facility Funds of the NSFC and CAS under Contracts No. U1532257, No. U1532258, and No. U1732263; CAS Key Research Program of Frontier Sciences under Contracts No. QYZDJ-SSW-SLH003 and No. QYZDJSSW-SLH040; 100 Talents Program of CAS; INPAC and Shanghai Key Laboratory for Particle Physics and Cosmology; German Research Foundation DFG under Contract No. Collaborative Research Center CRC 1044; Istituto Nazionale di Fisica Nucleare, Italy; Koninklijke Nederlandse Akademie van Wetenschappen (KNAW) under Contract No. 530-4CDP03; Ministry of Development of Turkey under Contract No. DPT2006K-120470; National Science and Technology fund; the Swedish Research Council; the Knut and Alice Wallenberg Foundation; U.S. Department of Energy under Contracts No. DEFG02-05ER41374, No. DE-SC-0010118, No. DE-SC0010504, and No. DE-SC-0012069; and University of Groningen (RuG) and the Helmholtzzentrum fuer Schwerionenforschung GmbH (GSI), Darmstadt.

\section{APPENDIX A: AMPLITUDES TESTED}

We list the amplitudes which are tested when searching for the nominal fit model but not used in the final result. Amplitudes with excited states $\left(m>1.0 \mathrm{GeV} / c^{2}\right)$ involved:

$$
\begin{aligned}
& D^{+} \rightarrow \bar{K}_{1}(1270)^{0} \pi^{+}, \bar{K}_{1}(1270)^{0} \rightarrow K_{S}^{0} \rho^{0}[D] . \\
& D^{+} \rightarrow \bar{K}_{1}(1270)^{0} \pi^{+}, \bar{K}_{1}(1270)^{0} \rightarrow K^{*-} \pi^{+}[S, D] . \\
& D^{+} \rightarrow K_{S}^{0} a_{2}(1320)^{+}, a_{2}(1320)^{+} \rightarrow \rho^{0} \pi^{+} \text {or }\left(\pi^{+} \pi^{-}\right)_{T} \pi^{+} . \\
& D^{+} \rightarrow K_{S}^{0} \pi(1300)^{+}, \pi(1300)^{+} \rightarrow \rho^{0} \pi^{+} \text {or }\left(\pi^{+} \pi^{-}\right)_{S} \pi^{+} . \\
& D^{+} \rightarrow K_{S}^{0} a_{1}(1640)^{+}, a_{1}(1640)^{+} \rightarrow \rho^{0} \pi^{+}[S, D] \text { or } \\
& \quad\left(\pi^{+} \pi^{-}\right)_{S} \pi^{+} . \\
& D^{+} \rightarrow \bar{K}(1460)^{0} \pi^{+}, \bar{K}(1460)^{0} \rightarrow\left(K_{S}^{0} \pi^{-}\right)_{S} \pi^{+} . \\
& D^{+} \rightarrow \bar{K}_{2}(1580)^{0} \pi^{+}, \bar{K}_{2}(1580)^{0} \rightarrow K^{*-} \pi^{+} \text {or }\left(K_{S}^{0} \pi^{-}\right)_{T} \pi^{+} . \\
& D^{+} \rightarrow \bar{K}^{*}(1410)^{0} \pi^{+}, \bar{K}^{*}(1410)^{0} \rightarrow K^{*-} \pi^{+} \text {or } K_{S}^{0} \rho^{0} .
\end{aligned}
$$

Amplitudes with only $K^{*-}, \rho^{0}$ and $f_{0}(500)$ involved:

$$
\begin{aligned}
& D^{+} \rightarrow K^{*-}\left(\pi^{+} \pi^{-}\right)_{S} . \\
& D^{+} \rightarrow\left(K^{*-} \pi^{+}\right)_{P, V, A, T} \pi^{+} . \\
& D^{+} \rightarrow\left(K_{S}^{0} \rho^{0}\right)_{V, T} \pi^{+} . \\
& D^{+} \rightarrow K_{S}^{0}\left(\rho^{0} \pi^{+}\right)_{P, V, A, T} . \\
& D^{+} \rightarrow\left(K_{S}^{0} f_{0}(500)\right)_{P, A, T} \pi^{+} . \\
& D^{+} \rightarrow K_{S}^{0}\left(f_{0}(500) \pi^{+}\right)_{P, A, T} .
\end{aligned}
$$


TABLE VIII. Interference of each amplitude, in units of $\%$ of total amplitude.

\begin{tabular}{|c|c|c|c|c|c|c|}
\hline & II & III & IV & $\mathrm{V}$ & VI & VII \\
\hline $\begin{array}{l}\text { I } \\
\text { II } \\
\text { III } \\
\text { IV } \\
\text { V } \\
\text { VI }\end{array}$ & $1.52 \pm 0.09$ & $\begin{array}{r}15.05 \pm 1.08 \\
0.41 \pm 0.06\end{array}$ & $\begin{array}{r}0.26 \pm 0.10 \\
-0.06 \pm 0.04 \\
-1.37 \pm 0.10\end{array}$ & $\begin{array}{r}-0.84 \pm 0.05 \\
0.03 \pm 0.01 \\
-0.07 \pm 0.03 \\
-2.00 \pm 0.11\end{array}$ & $\begin{array}{r}-2.72 \pm 0.18 \\
0.15 \pm 0.02 \\
-0.50 \pm 0.04 \\
-1 / 34 \pm 0.14 \\
0.34 \pm 0.04\end{array}$ & $\begin{array}{r}9.75 \pm 0.53 \\
0.15 \pm 0.03 \\
2.23 \pm 0.18 \\
1.42 \pm 0.19 \\
-0.89 \pm 0.05 \\
-4.00 \pm 0.25\end{array}$ \\
\hline & VIIII & IX & $X$ & XI & XII & XIII \\
\hline I & $-2.70 \pm 0.21$ & $0.64 \pm 1.04$ & $1.20 \pm 0.09$ & $-3.10 \pm 0.38$ & $-0.68 \pm 0.18$ & $1.67 \pm 0.14$ \\
\hline II & $-0.08 \pm 0.01$ & $0.04 \pm 0.04$ & $0.07 \pm 0.01$ & $-0.09 \pm 0.01$ & $-0.00 \pm 0.01$ & $-0.16 \pm 0.01$ \\
\hline III & $-0.71 \pm 0.06$ & $1.26 \pm 0.19$ & $0.08 \pm 0.08$ & $-3.20 \pm 0.20$ & $0.45 \pm 0.13$ & $-0.03 \pm 0.06$ \\
\hline IV & $-0.44 \pm 0.03$ & $-0.23 \pm 0.04$ & $6.19 \pm 0.84$ & $3.41 \pm 0.20$ & $0.01 \pm 0.00$ & $-4.20 \pm 0.68$ \\
\hline $\mathrm{V}$ & $0.04 \pm 0.00$ & $0.01 \pm 0.00$ & $-0.38 \pm 0.04$ & $0.54 \pm 0.03$ & $0.21 \pm 0.01$ & $0.28 \pm 0.02$ \\
\hline VI & $0.18 \pm 0.02$ & $0.19 \pm 0.01$ & $0.24 \pm 0.05$ & $1.50 \pm 0.10$ & $0.27 \pm 0.01$ & $-0.01 \pm 0.02$ \\
\hline VII & $0.51 \pm 0.09$ & $0.52 \pm 0.03$ & $0.36 \pm 0.11$ & $-1.18 \pm 0.20$ & $0.67 \pm 0.04$ & $0.79 \pm 0.08$ \\
\hline VIII & & $0.08 \pm 0.03$ & $-0.09 \pm 0.01$ & $0.56 \pm 0.03$ & $0.22 \pm 0.01$ & $0.41 \pm 0.03$ \\
\hline IX & & & $0.18 \pm 0.04$ & $-0.11 \pm 0.03$ & $0.64 \pm 0.12$ & $0.26 \pm 0.12$ \\
\hline $\mathrm{X}$ & & & & $0.96 \pm 0.23$ & $0.23 \pm 0.01$ & $-3.87 \pm 0.22$ \\
\hline XI & & & & & $0.88 \pm 0.05$ & $-0.38 \pm 0.18$ \\
\hline XII & & & & & & $0.20 \pm 0.01$ \\
\hline
\end{tabular}

Amplitudes without resonant state involved:

$$
\begin{aligned}
& D^{+} \rightarrow\left(K_{S}^{0}\left(\pi^{+} \pi^{-}\right)_{S}\right)_{P, A, T} \pi^{+} . \\
& D^{+} \rightarrow\left(K_{S}^{0}\left(\pi^{+} \pi^{-}\right)_{V}\right)_{P, V, A, T} \pi^{+} . \\
& D^{+} \rightarrow\left(K_{S}^{0}\left(\pi^{+} \pi^{-}\right)_{T}\right)_{A, T} \pi^{+} . \\
& D^{+} \rightarrow K_{S}^{0}\left(\left(\pi^{+} \pi^{-}\right)_{S} \pi^{+}\right)_{P, A, T} . \\
& D^{+} \rightarrow K_{S}^{0}\left(\left(\pi^{+} \pi^{-}\right)_{V} \pi^{+}\right)_{P, V, A, T} . \\
& D^{+} \rightarrow K_{S}^{0}\left(\left(\pi^{+} \pi^{-}\right)_{T} \pi^{+}\right)_{A, T} \\
& D^{+} \rightarrow\left(\left(K_{S}^{0} \pi^{-}\right)_{S \text {-wave }} \pi^{+}\right)_{A, T} \pi^{+} . \\
& D^{+} \rightarrow\left(\left(K_{S}^{0} \pi^{-}\right)_{V} \pi^{+}\right)_{P, V, A, T} \pi^{+} . \\
& D^{+} \rightarrow\left(\left(K_{S}^{0} \pi^{-}\right)_{T} \pi^{+}\right)_{A, T} \pi^{+} .
\end{aligned}
$$

Doubly Cabibbo-suppressed amplitudes:

$$
\begin{aligned}
& D^{+} \rightarrow K^{*+} \rho^{0} . \\
& D^{+} \rightarrow K_{1}(1270)^{0} \pi^{+}, K_{1}(1270)^{0} \rightarrow K^{*+} \pi^{-}[S, D] .
\end{aligned}
$$

\section{APPENDIX B: INTERFERENCE OF FIT FRACTION}

The interference between each amplitude is listed in Table VIII.
[1] H. Y. Cheng, Phys. Rev. D 67, 094007 (2003).

[2] H. Y. Cheng and C. W. Chiang, Phys. Rev. D 81, 074031 (2010).

[3] H. Y. Cheng, Phys. Lett. B 707, 116 (2012).

[4] M. Tanabashi et al. (Particle Data Group), Phys. Rev. D 98, 030001 (2018).

[5] B. S. Zou and D. V. Bugg, Eur. Phys. J. A 16, 537 (2003).

[6] M. Ablikim et al. (BESIII Collaboration), Nucl. Instrum. Methods Phys. Res., Sect. A 614, 345 (2010).

[7] M. Ablikim et al. (BESIII Collaboration), Chin. Phys. C 37, 123001 (2013).

[8] M. Ablikim et al. (BESIII Collaboration), Phys. Lett. B 753, 629 (2016).
[9] C. H. Yu et al., Proc. IPAC2016, Busan, Korea, 2016, https://dx.doi.org/10.18429/JACoW-IPAC2016-TUYA01.

[10] S. Agostinelli et al. (GEANT4 Collaboration), Nucl. Instrum. Methods Phys. Res., Sect. A 506, 250 (2003).

[11] S. Jadach, B. F. L. Ward, and Z. Was, Phys. Rev. D 63, 113009 (2001).

[12] E. Barberio and Z. Was, Comput. Phys. Commun. 79, 291 (1994).

[13] D. J. Lange, Nucl. Instrum. Methods Phys. Res., Sect. A 462, 152 (2001); R. G. Ping, Chin. Phys. C 32, 599 (2008).

[14] J. C. Chen, G. S. Huang, X. R. Qi, D. H. Zhang, and Y. S. Zhu Phys. Rev. D 62, 034003 (2000).

[15] M. Xu et al., Chin. Phys. C 33, 428 (2009). 
[16] H. Albrecht et al. (ARGUS Collaboration), Phys. Lett. B 340, 217 (1994).

[17] S. U. Chung, Phys. Rev. D 48, 1225 (1993); 57, 431 (1998); F. von Hippel and C. Quigg, Phys. Rev. D 5, 624 (1972).

[18] G. J. Gounaris and J. J. Sakurai, Phys. Rev. Lett. 21, 244 (1968).

[19] R. Aaij et al. (LHCb Collaboration), Phys. Rev. D 92, 032002 (2015).

[20] D. V. Bugg, Phys. Lett. B 572, 1 (2003); 595, 556(E) (2004).

[21] M. Ablikim et al. (BES Collaboration), Phys. Lett. B 598, 149 (2004).

[22] D. Aston et al. (LASS Collaboration), Nucl. Phys. B296, 493 (1988).

[23] B. Aubert et al. (BABAR Collaboration), Phys. Rev. D 78, 034023 (2008).
[24] M. Ablikim et al. (BESIII Collaboration), Phys. Rev. D 95, 072010 (2017).

[25] R. Aaij et al. (LHCb Collaboration), Eur. Phys. J. C 78, 443 (2018).

[26] D. Coffman et al. (Mark III Collaboration), Phys. Rev. D 45, 2196 (1992).

[27] M. Ablikim et al. (BESIII Collaboration), Phys. Rev. D 99, 092008 (2019).

[28] M. Williams, J. Instrum. 5, P09004 (2010).

Correction: The inline term appearing after Eq. (26) contained an error in the denominator and has been fixed. In Table III, the headings were misaligned and have been set right; the entry in the 11th line of the fourth column contained an error and has been fixed. 